\title{
Oncogenic Gata1 causes stage-specific megakaryocyte differentiation delay
}

\author{
Gaëtan Juban, ${ }^{1 a^{*}}$ Nathalie Sakakini, ${ }^{1,2 b *}$ Hedia Chagraoui, ${ }^{1}$ \\ David Cruz Hernandez, ${ }^{1,2}$ Qian Cheng, ${ }^{3}$ Kelly Soady, ${ }^{1,2}$ Bilyana Stoilova, ${ }^{1,2}$ \\ Catherine Garnett, ${ }^{1,2}$ Dominic Waithe ${ }^{3}$ Georg Otto, ${ }^{1 \mathrm{c}}$ Jessica Doondeea, ${ }^{1,2}$ \\ Batchimeg Usukhbayar, ${ }^{1,2}$ Elena Karkoulia, ${ }^{4}$ Maria Alexiou, ${ }^{5 \mathrm{~d}}$ \\ John Strouboulis, ${ }^{4 \mathrm{e}}$ Edward Morrissey, ${ }^{5}$ Irene Roberts, ${ }^{1,2,6}$ \\ Catherine Porcher $^{1 \#}$ and Paresh Vyas ${ }^{1,2,7 \#}$
}

Haematologica 2021

Volume 106(4):1106-1119

${ }^{1}$ MRC Molecular Hematology Unit WIMM, University of Oxford, Oxford, UK; ${ }^{2}$ Haematology Theme Oxford Biomedical Research Center, Oxford, UK; ${ }^{3}$ Center for Computational Biology WIMM, University of Oxford, Oxford, UK; ${ }^{4}$ Institute of Molecular Biology and Biotechnology, Foundation of Research \& Technology-Hellas, Heraklion, Crete Greece; ${ }^{5}$ Biomedical Sciences Research Center "Alexander Fleming", Vari, Greece; ${ }^{6}$ Department of Pediatrics University of Oxford, Oxford, UK and ${ }^{7}$ Department of Hematology, Oxford University Hospitals NHS Foundation Trust, Oxford, UK

${ }^{*}$ GJ and NS contributed equally as co-first authors.

${ }^{*} \mathrm{CP}$ and $\mathrm{PV}$ contributed euqally as co-senior authors.

aCurrent address: Institut NeuroMyoGène, Université Claude Bernard Lyon 1, CNRS UMR 5310, INSERM U1217, Université Lyon, Lyon, France.

${ }^{b}$ Current address: Cambridge Stem Cell Institute, Cambridge, UK.

${ }^{\circ}$ Current address: Genetics and Genomic Medicine, University College London Institute of Child Health, London, UK.

${ }^{d}$ Current address: Department of Dentistry, University of Alberta, Edmonton, Alberta, Canada.

eCurrent address: Rayne Institute School of Cancer \& Pharmaceutical Sciences, King's College London, London, UK

\section{Correspondence: \\ PARESH VYAS \\ paresh.yyas@imm.ox.ac.uk \\ GAËTAN JUBAN \\ gaetan.juban@univ-lyon1.fr}

Received: December 8, 2019.

Accepted: May 20, 2020.

Pre-published: June 11, 2020

https://doi.org/10.3324/haematol.2019.244541

(C)2021 Ferrata Storti Foundation

Material published in Haematologica is covered by copyright. All rights are reserved to the Ferrata Storti Foundation. Use of published material is allowed under the following terms and conditions:

https://creativecommons.org/licenses/by-nc/4.0/legalcode. Copies of published material are allowed for personal or internal use. Sharing published material for non-commercial purposes is subject to the following conditions:

https://creativecommons.org/licenses/by-nc/4.0/legalcode, sect. 3. Reproducing and sharing published material for commercial purposes is not allowed without permission in writing from the publisher.

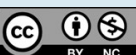

ABSTRACT

he megakaryocyte/erythroid transient myeloproliferative disorder (TMD) in newborns with Down syndrome (DS) occurs when $\mathrm{N}$ terminal truncating mutations of the hemopoietic transcription factor GATA1, that produce GATA1short protein (GATA1s), are acquired early in development. Prior work has shown that murine GATA1s, by itself, causes a transient yolk sac myeloproliferative disorder. However, it is unclear where in the hemopoietic cellular hierarchy GATA1s exerts its effects to produce this myeloproliferative state. Here, through a detailed examination of hemopoiesis from murine GATA1s embryonic stem cells (ESC) and GATA1s embryos we define defects in erythroid and megakaryocytic differentiation that occur late in hemopoiesis. GATA1s causes an arrest late in erythroid differentiation in vivo, and even more profoundly in ESC-derived cultures, with a marked reduction of Ter-119 cells and reduced erythroid gene expression. In megakaryopoiesis, GATA1s causes a differentiation delay at a specific stage, with accumulation of immature, kit-expressing $\mathrm{CD} 41^{\text {hi }}$ megakaryocytic cells. In this specific megakaryocytic compartment, there are increased numbers of GATA1s cells in S-phase of the cell cycle and a reduced number of apoptotic cells compared to GATA1 cells in the same cell compartment. There is also a delay in maturation of these immature GATA1s megakaryocytic lineage cells compared to GATA1 cells at the same stage of differentiation. Finally, even when GATA1s megakaryocytic cells mature, they mature aberrantly with altered megakaryocyte-specific gene expression and activity of the mature megakaryocyte enzyme, acetylcholinesterase. These studies pinpoint the hemopoietic compartment where GATA1s megakaryocyte myeloproliferation occurs, defining where molecular studies should now be focused to understand the oncogenic action of GATA1s. 


\section{Introduction}

The X-chromosome-encoded hematopoietic transcription factor GATA1 is essential for normal erythroid and megakaryocytic differentiation. ${ }^{1-3}$ Clonal mutations acquired in fetal life, leading to loss of the N-terminal 84 amino acids of GATA1, occur in approximately $28 \%$ of newborns with Downs syndrome (DS) and are associated with either a clinically overt, or clinically silent, myeloproliferative disorder known as transient myeloproliferative disorder (TMD) ${ }^{4.7}$ The mutant truncated GATA1 protein is known as GATA1short or GATA1s. In most neonates with DS the mutant fetal GATA1s clone disappears by 3 months of age (and Roberts and Vyas unpublished data). In approximately $3 \%$ of all neonates the TMD clone acquires additional mutations $s^{8,9}$ that transform the clone resulting in megakaryoblast-erythroid leukemia known as myeloid leukemia of Down syndrome (ML-DS). Germline mutations resulting in GATA1s, in disomic individuals and families also cause disease, but rather than being oncogenic cause cytopenia ${ }^{10}$ including the clinical phenotype of DiamondBlackfan anemia. ${ }^{11}$

In order to begin to understand how GATA1s perturbs hemopoiesis, a mouse model of GATA1s has been studied. ${ }^{12}$ These mice develop a transient megakaryoblastic myeloproliferative disorder that resolves in utero and likely originates from yolk sac hemopoiesis. Interestingly, these mice are anemic in utero leading to embryonic loss. Mice that survive then have minimal hemopoietic defects in adult life. Consistent with this human induced pluripotent stem cells (iPSC) derived from GATA1s-expressing TMD cells failed to complete erythropoiesis. ${ }^{13}$

This suggests that the N-terminal of GATA1 has a specific developmental role in restraining megakaryocyte production and is required for terminal red cell maturation. However, it is unclear which developmental hemopoietic cell populations require the $\mathrm{N}$-terminus of GATA1 and the cellular and molecular mechanisms responsible for perturbed hemopoiesis in GATA1s cells.

In order to identify the cellular populations most perturbed by GATA1s, we studied hemopoietic differentiation from both ESC culture-derived embryoid bodies (that recapitulate yolk sac hemopoiesis) and murine yolk sacs in GATA1s and control wild-type GATA1 mice. We define specific stages in megakaryocyte maturation, where GATA1s megakaryocytic cells are significantly increased in overall number, exhibit decreased apoptosis, have increased numbers of cells in S-phase, exhibit a delay in terminal maturation and mature abnormally. Importantly, this population affected by GATA1s mutations is also observed in human TMD samples.

\section{Methods}

Creation of gene targeted embryonic stem cells (ESC), growth and differentiation of murine ESC, characterisation of ESC, flow cytometry, gene expression analysis, cell staining and microscopy, acetylcholinesterase staining quantitation, cell cycle and apoptosis assays

Details are stated in the Online Supplementary Appendix. Antibody clones and colours are listed in the Online Supplementary Table S1. Raw RNA sequencing data have been deposited in Arrayexpress (https://WwW.ebi.ac.uk/arrayexpress) with accession number E-MTAB-8968. Western blotting was performed as previously described. ${ }^{14}$

\section{Mice}

Animal studies were approved by the University of Oxford's Ethics Committee and conducted in accordance with the UK Home Office regulations (PPL n'PA7C92A40). Embryos were processed as set out in the Online Supplementary Appendix.

\section{Human samples}

Parents gave written informed consent in accordance with the Declaration of Helsinki, and the study was approved by the Thames Valley Research Ethics Committee (06MRE12-10; NIHR portfolio no. 6362).

\section{Statistical analyses}

All experiments were performed using at least three different cultures or animals in independent experiments. The Student's $t$-test was used for statistical analyses. $P<0.05$ was considered significant.

\section{Results}

\section{Differentiation of bioGATA1 (bioG1) and bioGATA1s (bioG1s) cells}

Murine bioGata1 and bioGata1s alleles were created in male BirA ligase-expressing ESC ${ }^{15}$ by gene targeting of Xchromosome encoded Gata1 (Online Supplementary Figure $1 A-B)$. Correct targeting was verified by Southern blot analysis (Online Supplementary Figure 1C) and PCR (Online Supplementary Figure 1D-E). We generated three ESC types: BirA-bioGATA1s (hereafter, bioG1s) and as controls parental BirA (hereafter, BirA) and BirA-bioGATA1 (hereafter, bioG1).

In order to study the mGATA1s megakaryocyte phenotype, we used a 12 day megakaryocyte in vitro ESC differentiation protoco ${ }^{16}$ (Figure 1A). ESC were differentiated into embryoid bodies (EB), EB disaggregated at day 6 (d6), then $\mathrm{CD} 41^{+}$hemopoietic cells isolated by bead-enrichment and $\mathrm{kit}^{\mathrm{th}} \mathrm{CD} 41^{+}$cells fluorescence-activated cell sorting (FACS)-purified (Online Supplementary Figure S1F-G) for further 6-day culture on OP9 stromal cells with cytokines to promote megakaryocyte differentiation. Western blot analysis of d6 CD $41^{+}$cells confirmed bioG1 cells expressed only a single higher molecular weight fulllength bioGATA1 isoform, whereas bioG1s cells only expressed a single lower weight bioGATA1s isoform (Figure 1B). We next confirmed expression of Gata1 exon 3 (common to both Gata1 and Gata1s) in BirA, bioG1 and bioG1s cells and appropriately detected cDNA spanning Gata1 exon 2-3 only in BirA and bioG1 and not bioG1s cells (Figure 1C).

Next, we tested the lineage characteristics of cells produced by the 12 day culture. First, we took all cells at day 12 (d12) and confirmed expression of megakaryocyte genes gpIIB, gpVI, mpl and p-selectin in BirA, bioG1 and bioG1s cells but not in ESC (Figure 1C). Next, by staining d12 cells with megakaryocyte-specific acetylcholinesterase stain (Figure 1D) we confirmed megakaryocyte production. Interestingly, bioG1s cultures produced significantly fewer megakaryocytes providing a first clue that megakaryocyte differentiation is impaired by GATA1s.

In order to obtain a more complete initial view of megakaryocyte differentiation we analysed kit (marker of 
A

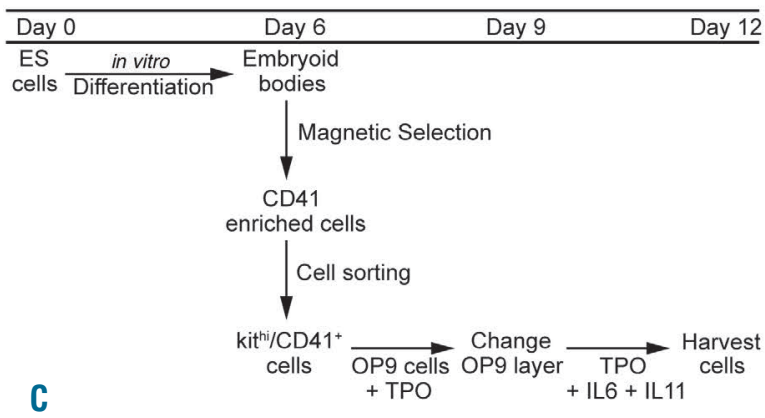

B

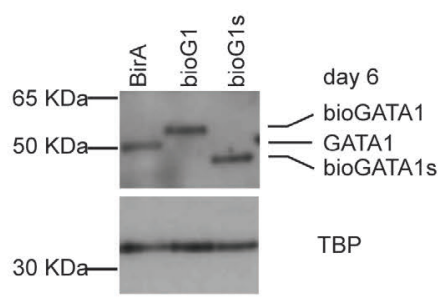

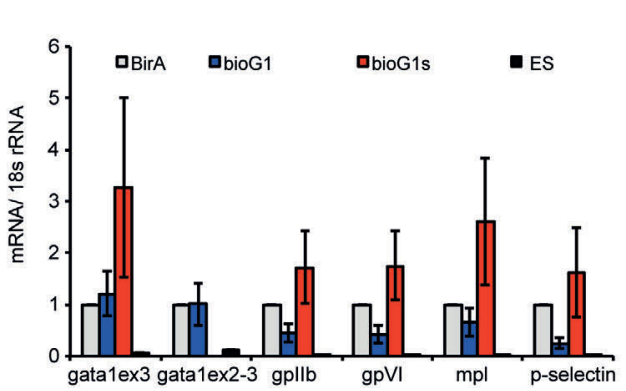

D
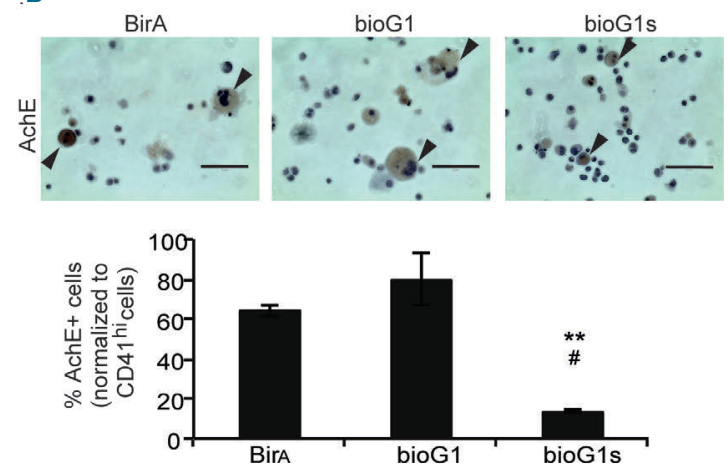

G

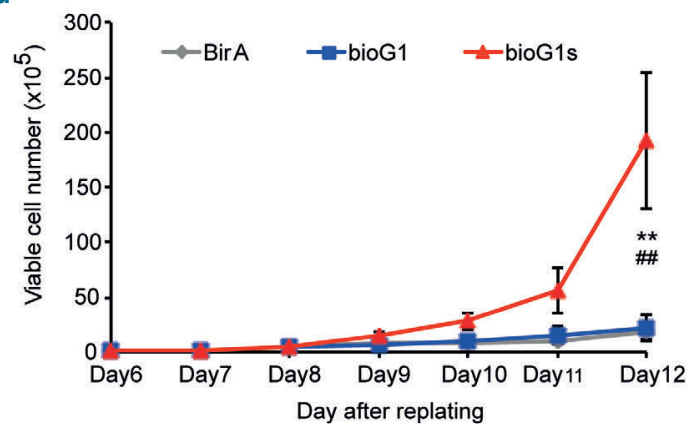

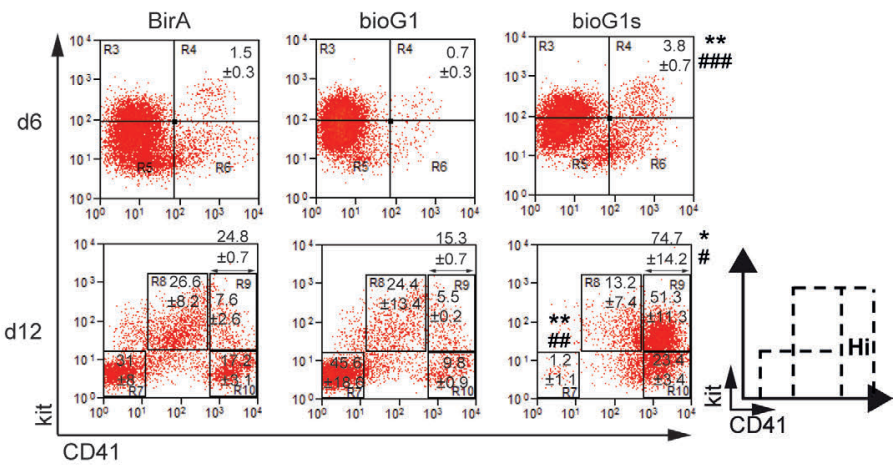

F
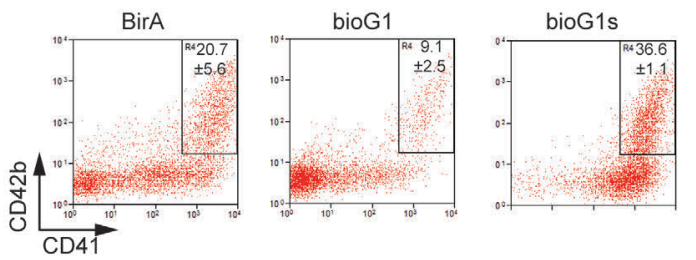

d12
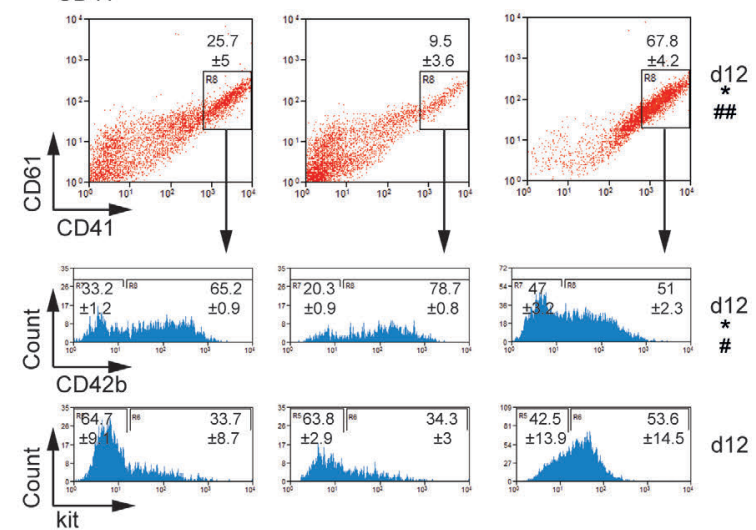

Figure 1. Gata1s embryonic stem cell-derived hematopoietic progenitors generate more immature megakaryocytes. (A) Protocol of in vitro megakaryocyte differentiation from embryonic stem cells (ESC). Top, day of culture. Below, sequential steps in the culture. TPO: thrombopoietin; IL6: interleukin 6; IL11: interleukin 11. (B) Western blot probed with anti-mGATA1 antibody (top panel) and anti-TBP antibody (bottom panel) using nuclear extracts from day 6 (d6) CD41 ${ }^{+}$cells from in vitro cultures. Genotype of cells is indicated above the blot. (C) Expression analysis of indicated genes in three independent day 12 (d12) embryoid bodies (EB)-derived megakaryocyte cultures from BirA (grey bar), bioG1 (blue bar) and bioG1s (red bar) cells or from undifferentiated ESC (black bar). (D) Top, photomicrographs of acetylcholinesterase (AChE) stained megakaryocytes (arrows) from d12 of culture. Scale bars indicate $100 \mu \mathrm{m}$. Below, bar plot of percentages of AChE ${ }^{+}$cells (relative to $\mathrm{CD} 41^{\text {hi }}$ cells) in three different cultures. (E) Flow cytometry showing expression of kit and CD41 on cells produced at d6 (above) or d12 (below) of in vitro culture. Left, BirA cells, middle, bioG1 cells and right, bioG1s cells. Figures in each gate show the mean \pm 1 standard deviation (SD) percentage of cells within the gate (five independent experiments). Position of CD41 ${ }^{\text {hi }}$ cells is indicated on the right of the $\mathrm{d} 12$ plot. (F) Flow cytometry showing expression of CD42b and CD41 (top) and CD61 and CD41 (middle) at d12 of culture. Bottom, CD42b and kit expression in CD $41^{+} C D 61^{+}$cells. Left, BirA cells, middle, bioG1 cells and right, bioG1s cells. Figures in each gate show the mean $\pm 1 S D$ percentage of cells within the gate (three independent experiments). (G) Viable cell count (y-axis) from d6 to $d 12$ in culture ( $x$-axis) when kith ${ }^{\text {hi }}$ 41 $1^{+}$cells from BirA (grey line), bioG1 (blue line) and bioG1s (red line) EB were replated on OP9 layer with cytokines (three independent experiments). Dead cells were excluded by trypan blue staining. ${ }^{*} P<0.05$ and ${ }^{*} * P<0.01$ vs. BirA. ${ }^{\#} P<0.05,{ }^{\# \#} P<0.01$ and ${ }^{\# \#} P<0.001$ vs. bioG1. 
A
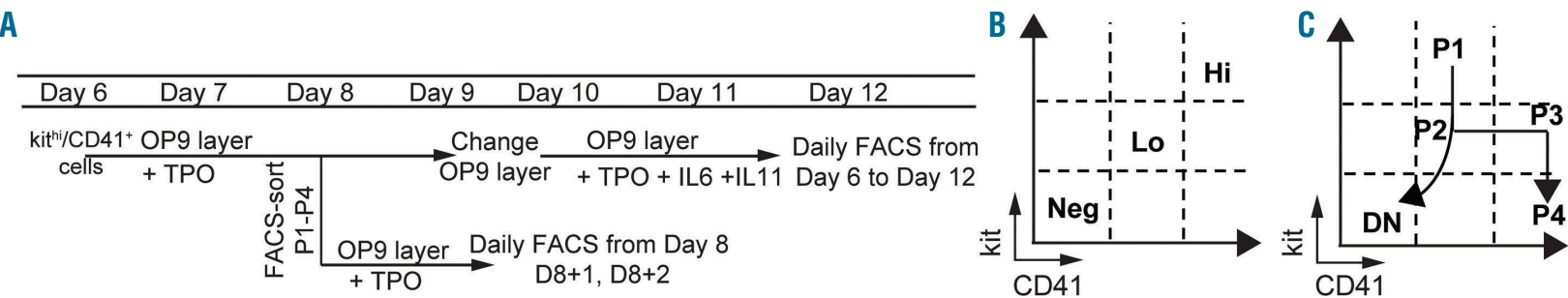

D
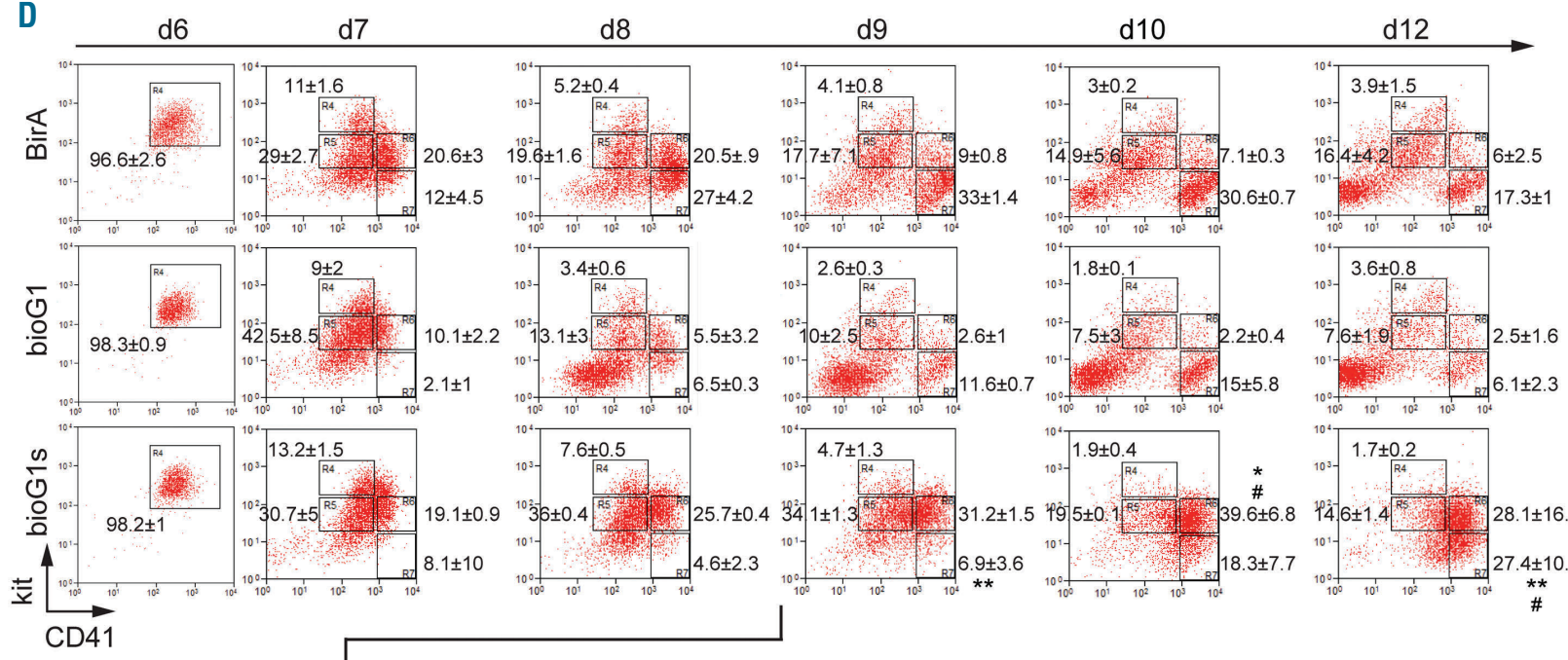

$104 \pm 0.2$

$E$
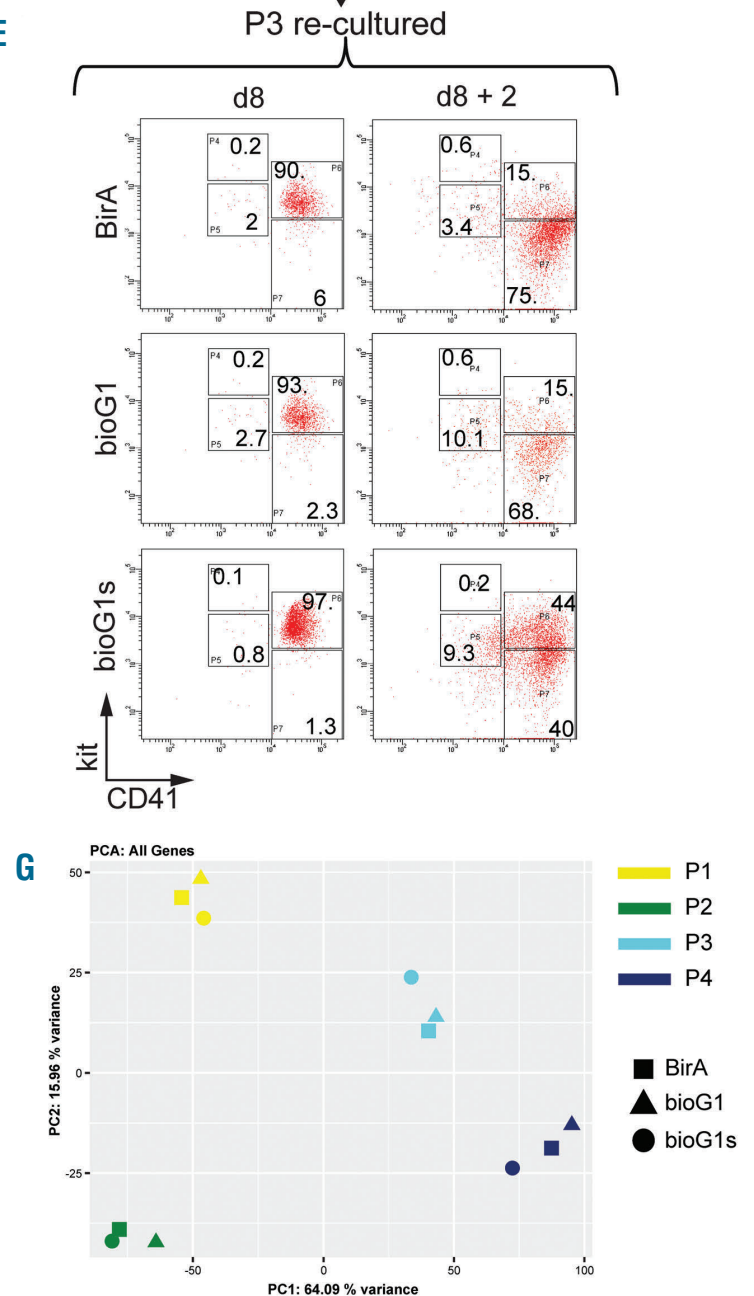

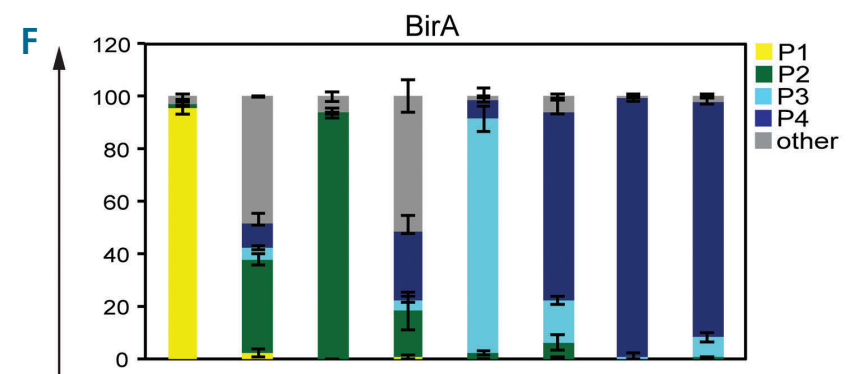

0
0
0
0
$\frac{\pi}{2}$
$\overline{0}$
0
0
0

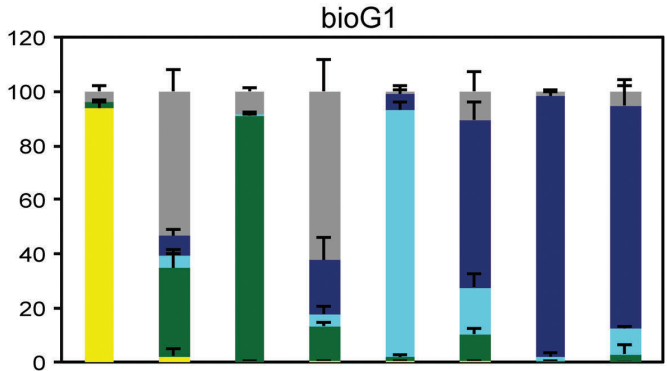

bioG1s

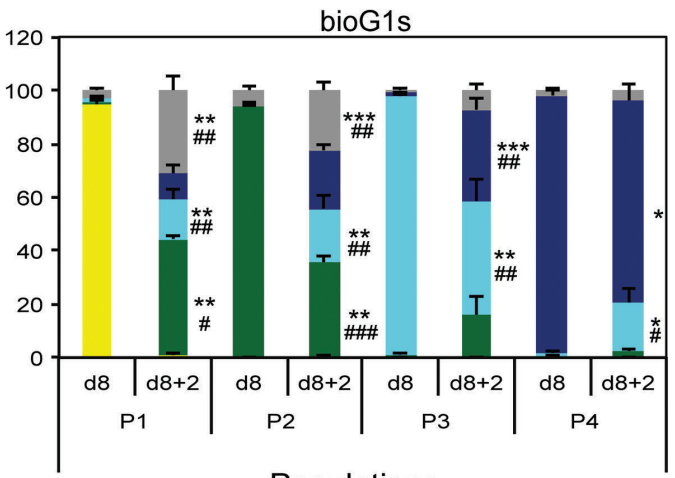

Populations

Figure 2. Legend on following page. 
Figure 2. (previous page) Gata1s hemopoietic cells have abnormal differentiation kinetics. (A) Schematic of experiment. Hemopoietic cells (kit ${ }^{\text {hi }} \mathrm{CD} 41^{10}$ ) from BirA, bioG1 and bioG1s day 6 (d6) embryoid bodies (EB) were cultured for another 6 days (up to day 12 [d12]). Aliquots of culture were analysed daily for kit and CD41 expression by fluorescence-activated cell sorting (FACS). In parallel, at d8, populations P1-P4 (see panel B-C) cells were purified by FACS-sorting, from cultures of three genotypes, re-cultured for 2 days and kit and CD41 analysed by FACS analysis. (B) Schematic of levels of kit and CD41 expression detected by flow cytometry. Neg: negative; lo: low and hi: high. Different levels of kit and CD41 expression define different hemopoietic cell populations in panels C-E. (C) Schematic summary of the data from panel (D), showing the two branches of hemopoietic differentiation undertaken by the initial kit ${ }^{\text {hi }} C D 41^{\text {lo }}$ (P1 population). $P 1$ cells differentiate into

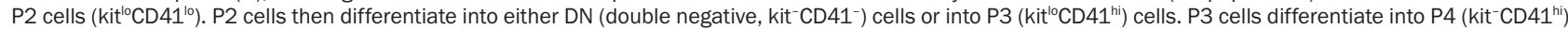
cells. (D) Representative FACS plots showing the differentiation of d6 hemopoietic cells (kit ${ }^{\text {hi }} C D 41^{10}$, termed P1 population) from BirA (top), bioG1 (middle) and bioG1s (bottom) cultures from d7 to d12 monitored by kit and CD41 expression. Numbers within gates are the mean percentage \pm 1 standard deviation of cells within the gate from three independent experiments. (E) Example of the re-culturing of FACS-purified d8 populations for two additional days. Here, P3 cells were FACS-purified from BirA cultures (top), bioG1 (middle) and bioG1s (bottom) cultures and re-cultured for 2 days. Left, FACS plots of post-sort purity checks of sorted P3 cell population. Right, expression of kit and CD41 expression after two days of culture. (F) Quantitation of the different populations generated by FACS-sorted d8 P1, P2, P3 and P4 populations after an additional 2 days of culture (three independent experiments for BirA and bioG1, four independent experiments for bioG1s). (G) Principal component analysis (PCA) plot showing P1 to P4 populations (each dot corresponds to the average of the four replicates) from each genotype using all genes analysed by RNA sequencing. Percentage variance for each PC is shown. ${ }^{*} P<0.05, * * P<0.01$ and $* * * P<0.001$ vs. BirA. ${ }^{\#} P<0.05,{ }^{\# \#} P<0.01$ and ${ }^{\# \#} P<0.001$ vs. bioG1.

immature hemopoietic cells) and CD41 (marker of megakaryocyte maturation) expression at $\mathrm{d} 6$ and $\mathrm{d} 12$ of culture (Figure 1E; Online Supplementary Figure S1F). D6 bioG1s EB produced significantly more $\mathrm{kit}^{+} \mathrm{CD} 41^{+}$(hemopoietic) cells. By d12, there were significantly more CD41 ${ }^{\text {hi }}$ cells in bioG1s cultures than bioG1 and BirA cultures but most bioG1s CD41 ${ }^{\text {hi }}$ cells still expressed the immaturity marker, kit. Finally, there were significantly fewer nonmegakaryocyte kitCD41 cells in bioG1s compared to bioG1 and BirA cultures.

In order to further characterize megakaryocyte marker expression, we confirmed that $\mathrm{CD} 41^{+}$megakaryocytes also co-expressed the mature megakaryocyte markers CD42b and CD61 at d12 (Figure 1F; Online Supplementary Figure $S 1 H)$, paradoxically even in kit expressing cells in bioG1s cells. Interestingly, there were significantly greater percentage of $\mathrm{CD} 41^{+} \mathrm{CD} 61^{+}$cells in bioG1s cultures compared to control bioG1 and BirA cultures. Finally, bioG1s cells also expressed lower levels of the maturity marker CD42b on CD $41^{+} \mathrm{CD} 61^{+}$cells.

Finally, we measured cell growth by counting viable cell numbers daily from d6 to d12 (Figure 1G). Numbers of cells in BirA, bioG1 and bioG1s were similar from d6 to d9 but then increased significantly in bioG1s cultures and were 10-fold greater at d12 compared to both BirA and bioG1 cultures.

In summary, the cultures produced both megakaryocyte and non-megakaryocyte cells. Compared to wild-type GATA1 hemopoietic cells, bioG1s cells were more proliferative, producing more immature megakaryocytes and fewer non-megakaryocytic cells.

In order to characterize the kinetics of abnormal differentiation we sampled cultures daily from d6 to d12 (Figure 2A-D). Starting with FACS-purified d6 $\mathrm{kit}^{\mathrm{hi}} \mathrm{CD} 41^{\text {lo }}$ cell population (termed P1), we monitored maturation (lower the level of kit expression the more mature the cells) and acquisition of the megakaryocyte lineage (increasing CD41 expression).

The temporal sequence of flow cytometric plots suggested that control cells (BirA and bioG1) first showed a decrease in kit expression level, generating a kit $^{\text {lo }} \mathrm{CD} 41^{\text {lo }}$ population (termed P2) (seen at d7). Cells in P2 then divided into two differentiation branches (Figure 2B-D; Online Supplementary Figures S2A, S3A). In one branch, cells progressively lost expression of both kit and CD41 (d8 onwards) to generate a $\mathrm{kit}^{-} \mathrm{CD} 41^{-}$population (double negative $[\mathrm{DN}]$ cells). This DN population was mainly composed of erythroid cells (see below). In the other branch, P2 cells also differentiated towards the megakaryocytic lineage with an increase in CD41 expression level (kit ${ }^{\text {lo }} \mathrm{CD} 41^{\mathrm{hi}}$ population, called P3) (d7 onwards) followed by loss of kit expression (kit ${ }^{-} \mathrm{CD} 41^{\text {hi }}$ population, called $\left.\mathrm{P} 4\right)$ (d8 onwards). In contrast, there were two marked differences in bioG1s cultures (Figure 2D). First, they generated far fewer DN cells. Second, bioG1s cells showed enhanced differentiation into the P3 population (d9-10) but with a delay of differentiation into P4 (best seen at d8-9, more cells in P4 in control cultures). In contrast, there were more cells in bioG1s in $\mathrm{P} 4$ at $\mathrm{d} 12$.

Though this temporal analysis was suggestive of two differentiation branches and hierarchical relationships between P2, P3, P4 and P2 and DN (Figure 2C), in order to provide more definitive proof we FACS-sorted each population (P1, P2, P3, P4) individually at d8 and re-cultured them for 2 days. During re-culture we analyzed kit and CD41 expression in the progeny produced (Figure 2E, reculture of P3, Figure 2F, data summary; Online Supplementary Figures S2B, S3B). FACS-sorted P1 generated all the other populations. Purified P2 generated all the populations except P1. P3 differentiated primarily into P3 and P4 only but not DN cells. Finally, re-culturing of P4 cells generated principally $\mathrm{P} 4$ cells. These data were consistent with the differentiation branches and hierarchical relationships in Figure 2C. Finally, we performed RNAseq on sorted populations and showed that they were transcriptionally distinct. A PCA plot using all expressed genes revealed that each population form a separate cluster, regardless of their genotype. Moreover P3 and P4 populations segregated along PC1, suggesting they related to each other (Figure $2 \mathrm{G})$. Analyses have also been run using the top most variant genes across all populations, ranging from 100 to 10,000 genes and revealed a similar pattern (Online Supplementary Figure S3C).

Comparing the differentiation potential of FACS-sorted bioG1s populations to control BirA and bioG1 cells (Figure 2F), bioG1s P1 cells also generated significantly more P2 and P3 cells than BirA and bioG1 P1 cells. BioG1s P2 cells also generated significantly more P2 and P3 cells than BirA and bioG1 P2 cells. Finally, bioG1s P3 cells generated more P3 but fewer P4 cells compared to BirA and bioG1 P3 cells.

Taken together, $\mathrm{kit}^{\text {hi }} \mathrm{CD} 41^{\text {lo }}$ hemopoietic progenitors differentiate either into non-megakaryocytic DN cells or megakaryocytic cells with increased CD41 expression and loss of kit expression. BioG1s mutant cells have a reduced ability to differentiate into DN cells but generate more megakaryocytic cells but with a partial differentiation delay at the P3 population stage.

\section{Reduced erythroid differentiation by bioGATA1s hemopoietic cells}

In order to confirm the identity of $\mathrm{kit}^{-} \mathrm{CD} 41^{-}$cells $(\mathrm{DN})$, we analyzed morphology, cell surface markers and mRNA 
A

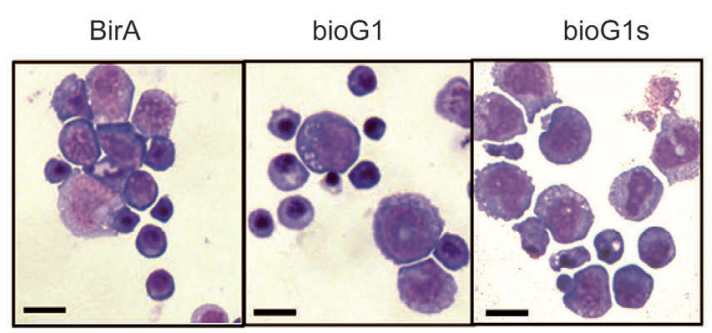

B 14000 -Ter119+ $\quad$ Mac1+ and/or Gr1+ $\stackrel{\text { Mer119-I }}{\text { Mac1-/Gr1- }}$

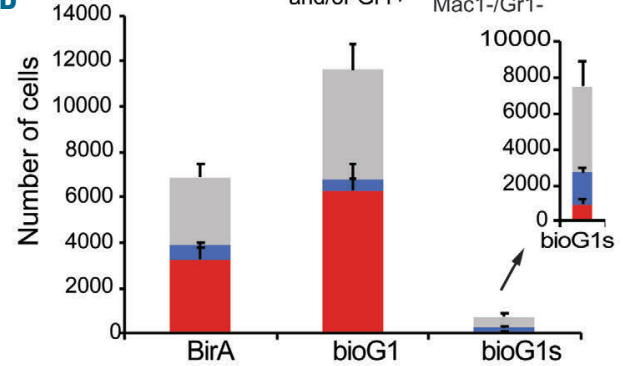

C

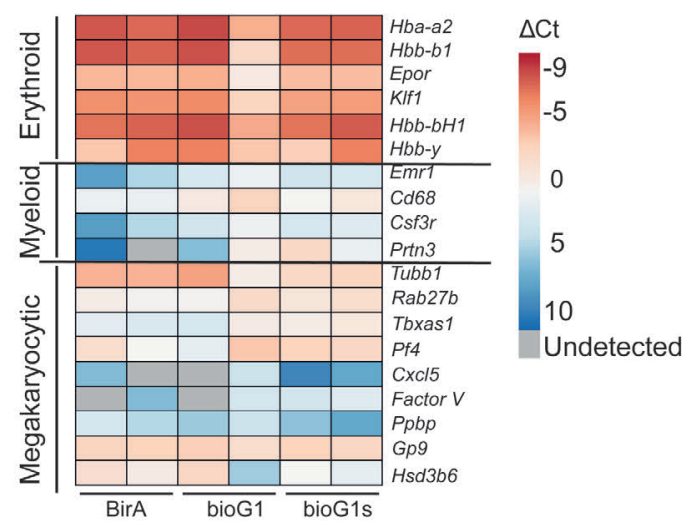

D
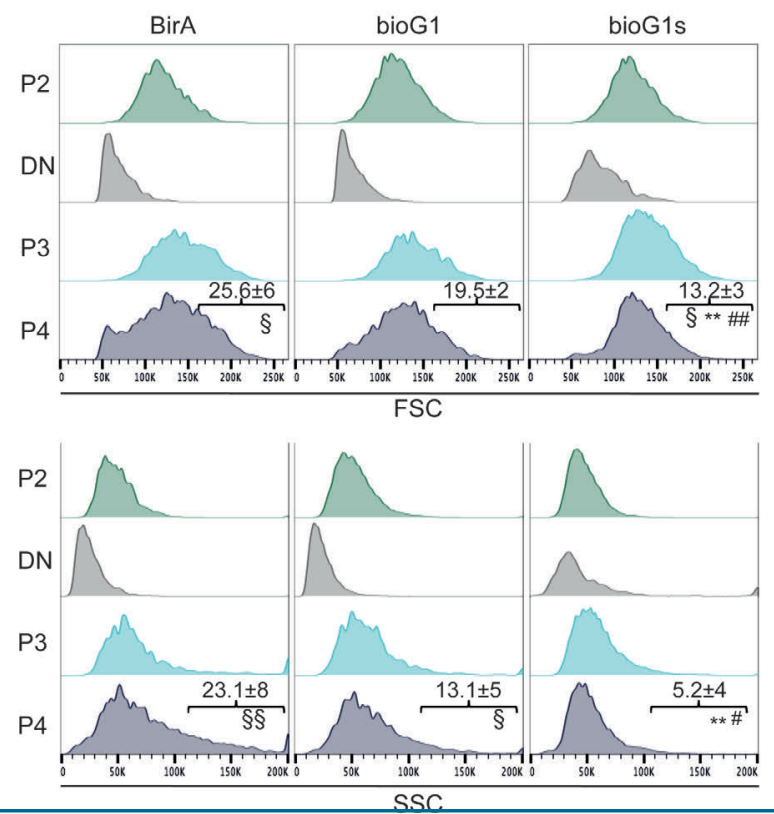

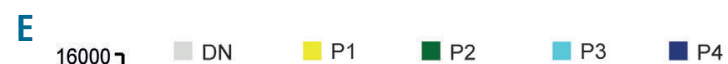
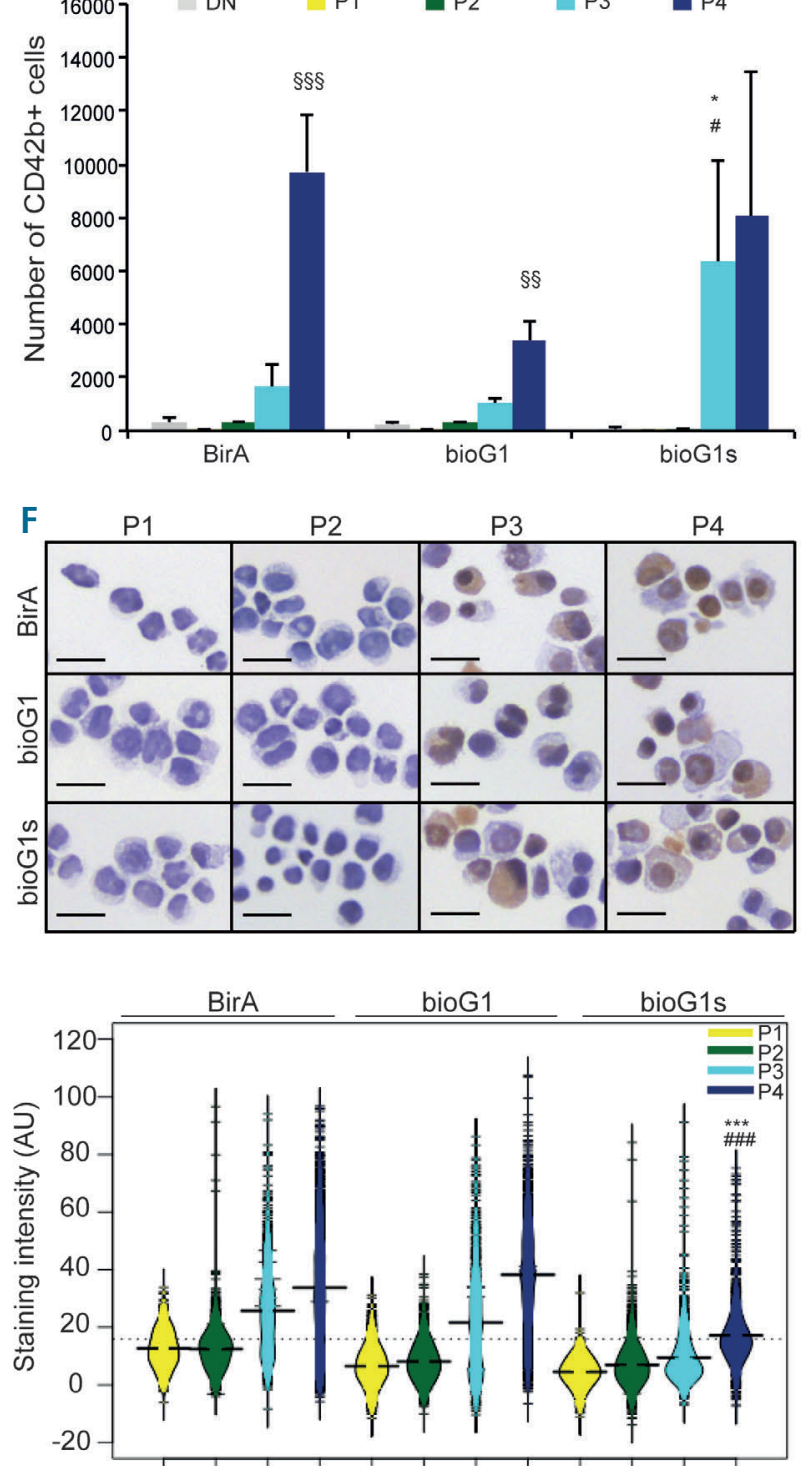

G

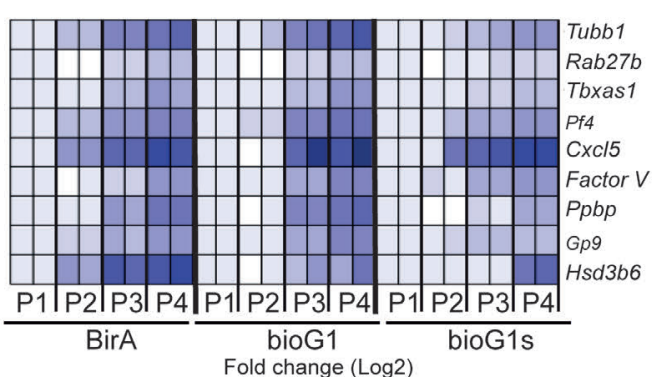

H

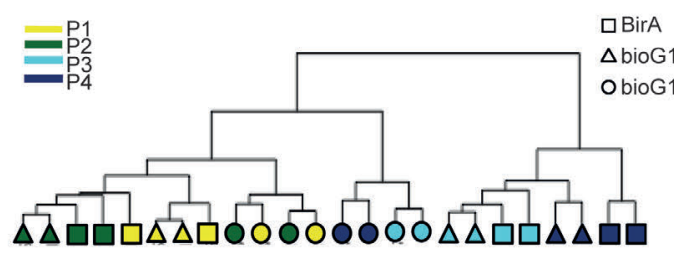

Figure 3. Legend on following page. 
Figure 3. (previous page) Gata1s cells fail to produce erythroid cells and mature megakaryocytes. (A) Micrographs of May-Grunwald-Giemsa-stained cytospins of fluorescence-activated cell sorting (FACS)-purified double negative (DN) (kit ${ }^{-}$CD41-) cells at day 10 (d10). Genotype of cells is indicated above. Scale bars represent $25 \mathrm{um}$. (B) Bar plot of number of erythroid cells (Ter119+) and myeloid cells (Gr1 ${ }^{+}$and/or Mac1 ${ }^{+}$) within the DN population at d10 of culture in BirA, bioG1 and bioG1s cultures. 250,000 total cells analysed by FACS in each case. (C) Heatmap of mRNA expression of selected erythroid (top), myeloid (middle) and megakaryocytic (bottom) genes (in rows) in DN BirA (left), bioG1 (middle) and bioG1 (right) cells at d10. Data from two independent biological replicates is shown. (D) Representative histogram (three independent experiments were performed) showing size (forward scatter [FSC-A], top panel) and granularity (side scatter [SSC-A], bottom panel) assessed by flow cytometry. Data from BirA (left), bioG1 (middle), bioG1s (right) from P2, DN, P3, P4 populations is shown. In P4, numbers indicate the mean percentage \pm 1 standard deviation (SD) of cells within the gate. (E) Bar plot showing the number of cells expressing CD42b at d12 from DN, P1, P2, P3 and P4 populations from genotypes indicated (three independent experiments). (F) Top, representative micrographs of acetylcholinesterase (AChE) staining of FACS-purified P1 to P4 d10 BirA, bioG1 and bioG1s populations. Scale bars represent $10 \mu \mathrm{m}$. Bottom, quantitation of AChE staining, from 500 cells, analyzed from three independent experiments. Bean-plot of staining intensity expressed in arbitrary units (AU) (y-axis) for each population (P1 to P4) from BirA, bioG1 and bioG1s cells. (G) Heatmap showing the fold change in expression of selected megakaryocytic genes (indicated on the right) in FACS-purified P1, P2, P3 and P4 at d10 (columns). Data from two independent biological replicates is shown. Genotype of the cells is indicated below the heatmap. $(\mathrm{H})$ Hierarchical clustering using mRNA data from $(G)$. * $P<0.05$, ${ }^{*} * P<0.01$ and $* * * P<0.001$ between bioG1s and BirA. ${ }^{\#} P<0.05,{ }^{\#} P<0.01$ and ${ }^{\# \# \#} P<0.001$ between bioG1s and bioG1. ${ }^{\S} P<0.05$ and ${ }^{\S} P<0.01$ between $\mathrm{P} 4$ and $\mathrm{P} 2$. ${ }^{\$ \$} P<0.01$ and ${ }^{\$ \$ \$} P<0.001$ between $\mathrm{P} 4$ and $\mathrm{P} 3$.

expression profile of FACS-purified cells (Figure 3A-C; Online Supplementary Figure S4A-F). Morphologically, DN cells were primarily erythroid cells, at different stages of maturation, with hardly any granulated myeloid cells (Figure 3A). Approximately 50\% of BirA and bioG1 DN cells were Ter119+ and $5 \%$ were $\mathrm{Mac1}^{+}, \mathrm{Gr}^{+}$or both $\mathrm{Mac1}^{+} \mathrm{Gr1}^{+}$(Figure 3B; Online Supplementary Figure S4A-C). The DN population was markedly reduced in bioG1s cultures compared to bioG1 (24-fold) and BirA cultures (16fold) and with more myeloid than erythroid cells. In all three genotypes approximately $50-60 \%$ DN cells were Ter119-Gr1-Mac1-'. Given FACS-purified DN cells showed higher mRNA expression of erythroid genes and lower expression of myeloid and megakaryocytic genes (Figure 3C; Online Supplementary Figure S3D-F), one possible lineage assignment for the Ter119- $\mathrm{Gr}^{-} \mathrm{Mac1}^{-}$cells could be immature Ter119- erythroid cells.

\section{Altered megakaryocytic maturation of bioGATA1s cells}

During differentiation, megakaryocytes enlarge considerably, acquire granules and develop a demarcation membrane system for proplatelet formation. We used multiple approaches to study megakaryocyte maturation as cells progressed from P2 to P4. First, morphologically, cells in populations P1 and P2 were small, with a blast morphology (Online Supplementary Figure S5A). In contrast, cells in P3 and P4 were larger, particularly P4 which were maturing megakaryocytes. In order to quantify these changes, we measured cell size (forward scatter[ FSC-A]) and granularity (side scatter [SSC-A]) by flow cytometry (Figure 3D). Concordantly, there was a progressive increase of size and granularity from $\mathrm{P} 2$ to $\mathrm{P} 3$ and $\mathrm{P} 4$. A similar trend was also seen in the mutant cells, but to a lower extent. Closer inspection of FSC and SSC profiles showed a lower proportion of larger and more granular cells in the P4 population in bioG1s compared to control BirA and bioG1 populations. Finally, as expected the erythroid-dominant DN cells showed decreased cell size and granularity compared to P2 cells.

Next, we studied CD42b expression in DN, P1 to P4 populations at d12 (Figure 3E; Online Supplementary Figure $S 5 B-E)$. As expected, very few cells in DN, P1 and P2 expressed CD $42 \mathrm{~b}(<4 \%$; absolute numbers $<200$ cells). In contrast, and as expected, the absolute number (Figure $3 \mathrm{E}$ ) and proportion (Online Supplementary Figure $S 5 E$ ) of CD42 $\mathrm{b}^{+}$cells in P3 and P4 were significantly much higher than in DN, P1 and P2. Importantly, there were significant differences between bioG1s and BirA and bioG1. Absolute numbers of CD42 $b^{+}$in P3 (Figure 3E) were significantly greater in bioG1s compared to BirA and bioG1 supporting the hypothesis that compared to wild-type
GATA1, GATA1s promotes proliferation of kit immature megakaryocytes (P3). In contrast, the absolute numbers of mature kit ${ }^{-} \mathrm{CD} 42 \mathrm{~b}^{+} \mathrm{P} 4$ bioG1s cells were no different compared to bioG1 and BirA.

Furthermore, the absolute number and ratio of CD42b+ cells in P4 relative to P3 was greater in BirA and bioG1. In contrast, in bioG1s the absolute number and ratio of $\mathrm{CD}_{2} \mathrm{~b}^{+}$cells was not significantly different between P3 and P4 (Figure 3E; Online Supplementary Figure S5E). This supports the hypothesis that GATA1s, compared to wildtype GATA1, is less effective at driving maturation of P3 megakaryocytic cells to P4 megakaryocytic cells.

Next, we measured activity of acetylcholinesterase, an enzyme whose activity increases with megakaryocyte maturation, by quantitating intensity of an acetylcholinesterase driven cytochemical reaction in purified $\mathrm{P} 1$, P2, P3 and P4 populations (Figure 3F). The intensity of acetylcholinesterase-induced cytochemical staining was low in P1 and P2 and increased in P3 cells, and increased further, in P4 cells. Importantly, there was significantly lower cytochemical staining in P3 and P4 in bioG1s compared to control BirA and bioG1 cells, which may reflect the smaller size of P3 and P4 bioG1s cells and/or difference in maturation state of bioG1s cells.

Finally, we tested mRNA expression of megakaryocyte specific genes in P1-P4 in all three genotypes (Figure 3G; Online Supplementary Figure S4E-F). There was reduced expression of megakaryocyte genes in $\mathrm{P} 3$ and $\mathrm{P} 4$ in bioG1s compared to BirA and bioG1 cells (Tubb1, Factor V, Pbbp, Gp9 and Hsd3b6). Hierarchical clustering analysis confirmed that bioG1s P3 and P4 cells were transcriptionally more closely related to the more immature P1 and P2 cell populations than P3 and P4 from GATA1 wild-type cells (Figure 3H; Online Supplemental Figure S5F).

Taken together, these data confirm megakaryocytes mature from $\mathrm{P} 1$ to $\mathrm{P} 4$. BioG1s produce more immature megakaryocytes (P3) but they fail to differentiate as efficiently into the most mature megakaryocyte population (P4) compared to wild-type cells.

\section{Decreased apoptosis and increased proliferation in mutant P3 population}

In order to understand why the number of bioG1s cells increased during megakaryocytic differentiation (Figure $1 \mathrm{G}$; Figure $3 \mathrm{E}$ ), we asked if this was due to reduced apoptosis (Figure 4A-B) and/or increased cycling of cells (Figure 4C-D) in bioG1s compared to control BirA cells. We performed flow analyses in P1-P4 sub-populations at d8 (for apoptosis) or $\mathrm{d} 9$ (for cell cycle) (2 or 3 days after re-culture of $\left.\mathrm{kit}^{\mathrm{hi}} \mathrm{CD} 41^{\mathrm{lo}}\right)$. There was a significant, and specific, decrease in Annexin $\mathrm{V}^{+}$cells in bioG1s P3 cells. There was 
A
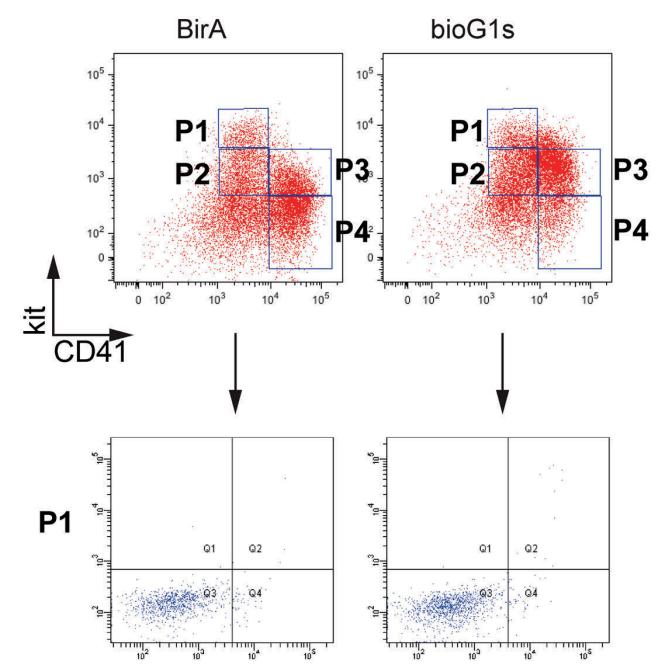

P2
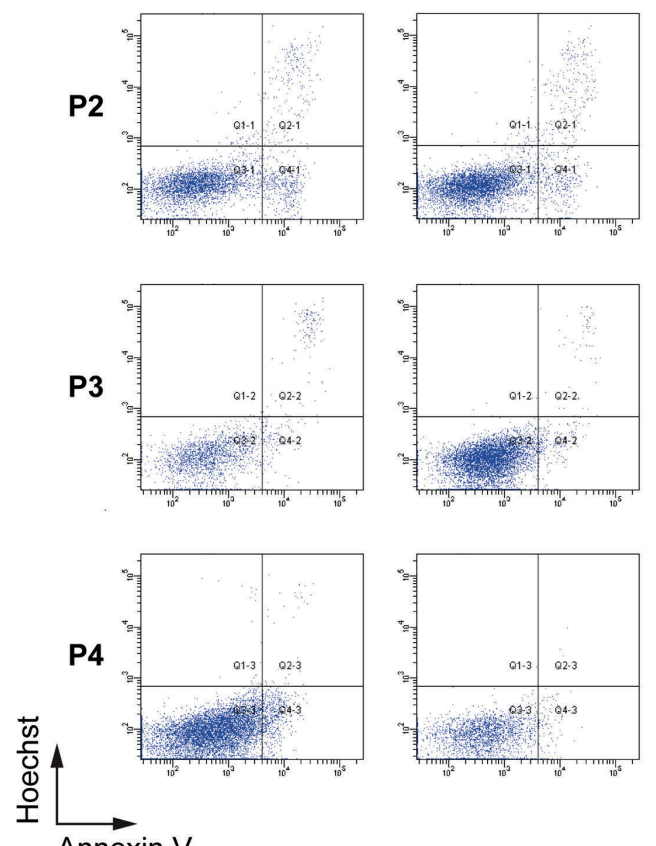

Annexin V

B
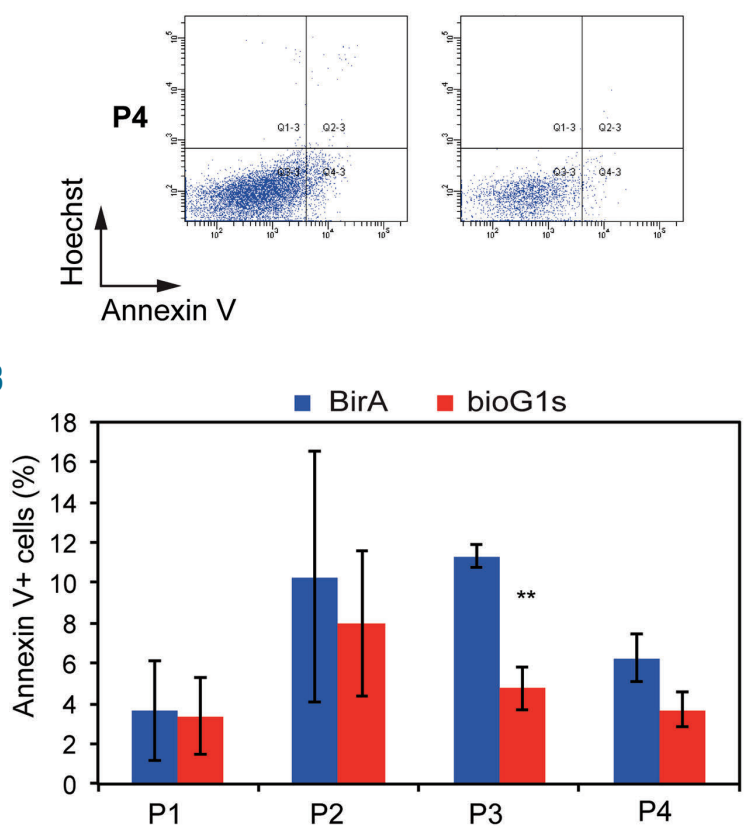

C
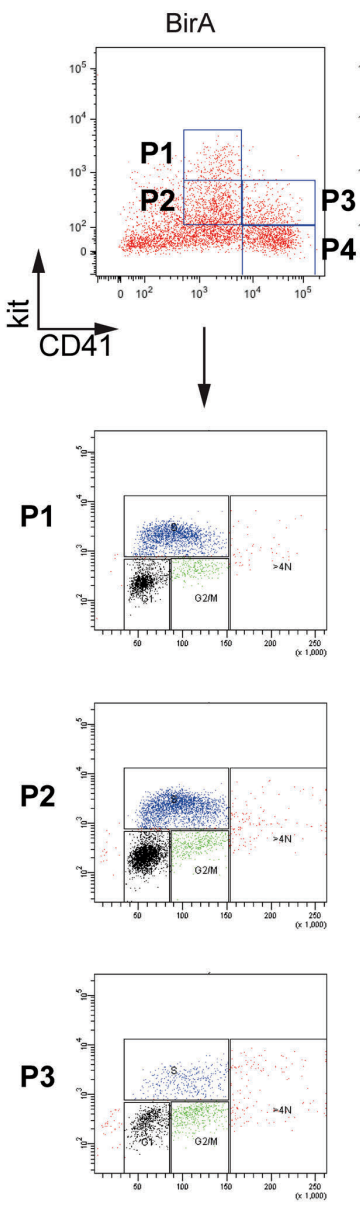
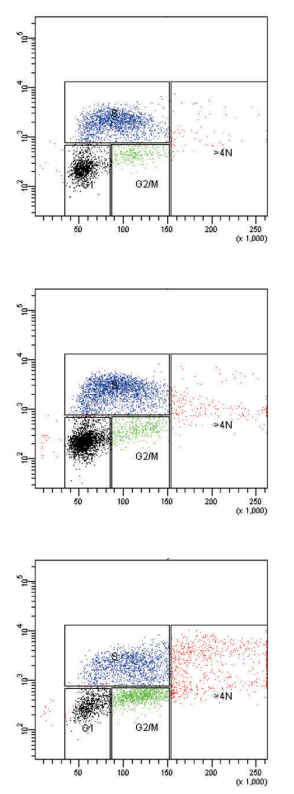

bioG1s
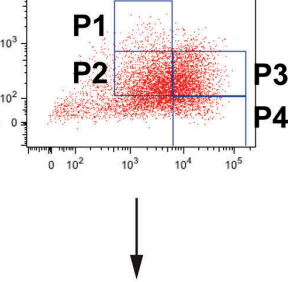

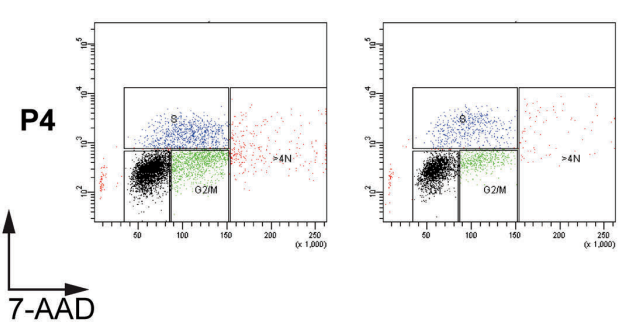

린

D

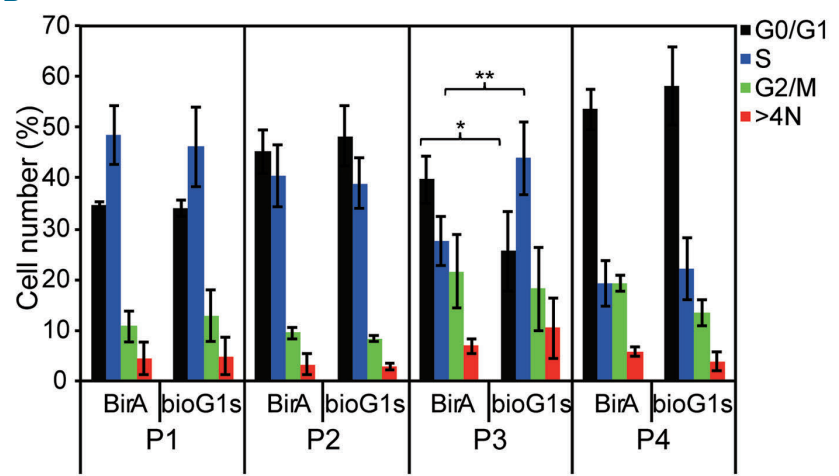

Figure 4. Increased proliferation and decreased apoptosis in GATA1s P3 cells. (A) Flow cytometry analysis, from one representative experiment (of three independent experiments) at day 8 (d8), showing kit and CD41 expression of BirA (left) and bioG1s (right) cells (top). P1 to P4 populations indicated. Below, Annexin V and Hoechst staining within P1 to P4 populations. (B) Bar plot of data from all three experiments showing mean percentage \pm 1 standard deviation (SD) of AnnexinV ${ }^{+}$cells in BirA and bioG1s cultures. (C) Representative flow cytometry analysis, from one experiment (of three independent experiments) at d9. Details as set out in (A). Below, cell cycle analysis determined by EdU incorporation and 7-AAD staining. (D) Bar plot from all three experiments showing mean percentage \pm 1 SD of cells in G0/G1, S, G2/M phases of cell cycle and cells with $>4 \mathrm{~N}$ ploidy, in BirA and bioG1s cultures. $* P<0.05$ and $* * P<0.01$ between bioG1s and BirA 

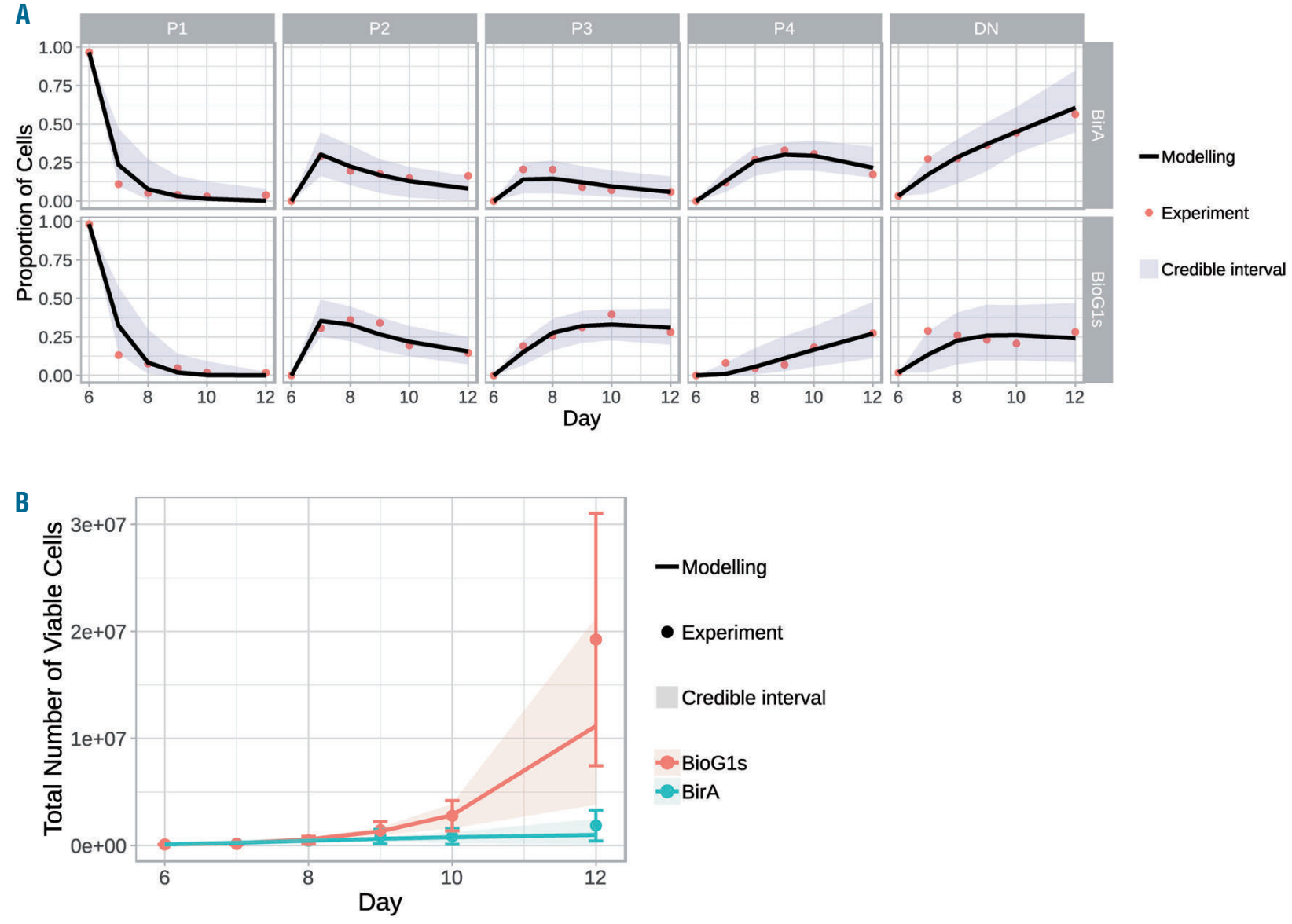

C
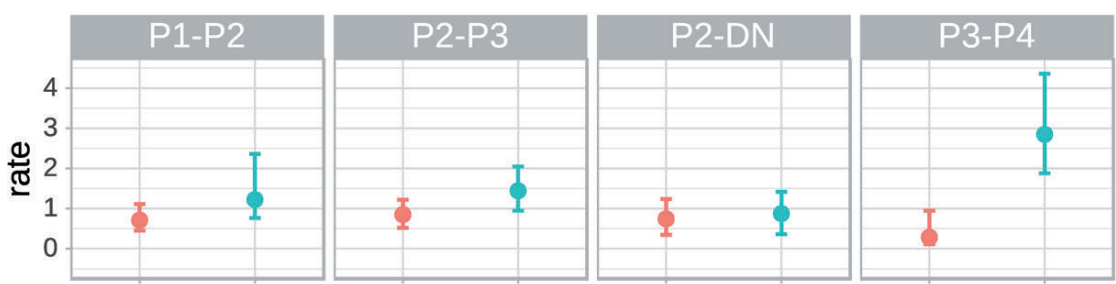

- BirA
- BioG1s

Figure 5. Cell fate modeling suggests GATA1s enhances cell division in P3 cells and reduces commitment to P4. (A) Fit of the mathematical model to the proportions of cells in each population (three independent experiments for BirA, four independent experiments for bioG1s). Top, BirA cells, below, bioG1s cells. The points are the data taken from Figure 2 and the solid line is the model fit. The shaded region is the 95\% Confidence Interval (Cl) for the data - i.e., 95\% of the data should lie within the shaded region. (B) Growth curve for the total number of cells modeled from the model fit. Top, BirA cells, below, bioG1S cells. Note the difference in scale of the $y$-axis. The error bars of the data are two-times the standard deviation of the replicates. Note the rate of transition of P3 to P4 is much lower for bioG1s. (C) Inferred cell transition rates between the populations and their $95 \% \mathrm{Cl}$.

also a specific, and significant, increase of bioG1s P3 cells in S-phase and decrease in G1/G0 phase.

\section{Modeling transitions through differentiation}

Using the kinetic data of differentiation (Figure 2), together with absolute cell numbers produced per initial cell numbers and the cell cycle and apoptosis data (Figure 4) we have developed a mathematical model (see the Online Supplementary Appendix) to study the rates at which the cells transition between $\mathrm{P} 1$ to all other populations and how Gata1s mutation alters the kinetics of transition. The fit of the model to the data can be seen in Figure $5 \mathrm{~A}$ and the modeled cell numbers in culture (Figure 5B) closely mirrors the actual cell numbers produced in culture (Figure $1 \mathrm{G})$. Comparing the rates of transition between the different populations, for BirA and bioG1s cells, only the rate of transition of P3 and P4 was different between BirA and bioG1s cells. Here, bioG1s showed statistically markedly reduced transition between $\mathrm{P} 3$ and P4 compared to BirA cells (Figure 5C). This slower transition from P3 to P4 produces an accumulation of cells in $\mathrm{P} 3$, where cells are proliferating more than in $\mathrm{P} 4$. This provides a likely explanation for the large increase in cell numbers seen in Figure 1G for bioG1s cells from d10 to d12.

\section{GATA1s phenotype is recapitulated in vivo}

Next, we asked if the in vitro EB-derived P1-P4 populations were present in mouse development. EB hemopoiesis mimics yolk sac hemopoiesis ${ }^{17,18}$ in that it first produces kit ${ }^{+} \mathrm{CD} 41^{+} \mathrm{CD} 16^{-} \mathrm{CD} 32^{-}$primitive yolk sac erythroid progenitors with myeloid and megakaryocytic potential, ${ }^{17,19}$ followed by $\mathrm{kit}^{+} \mathrm{CD} 41^{+} \mathrm{CD} 16^{+} 32^{+}$definitive ery- 
A
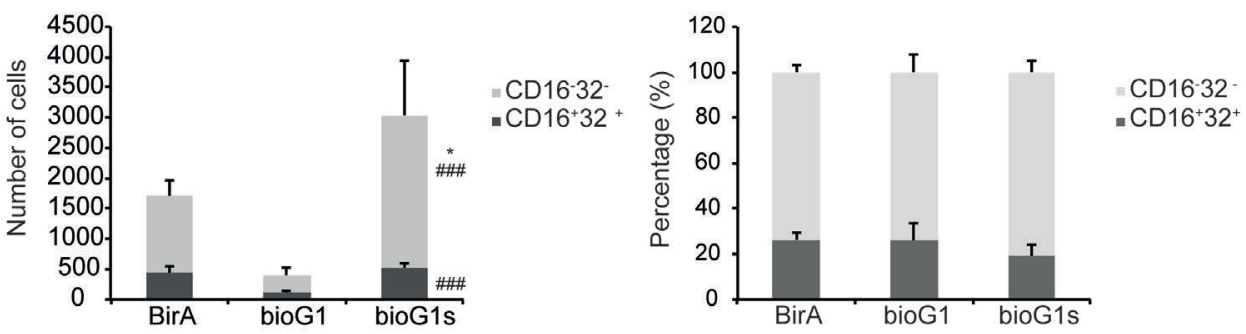

B

E
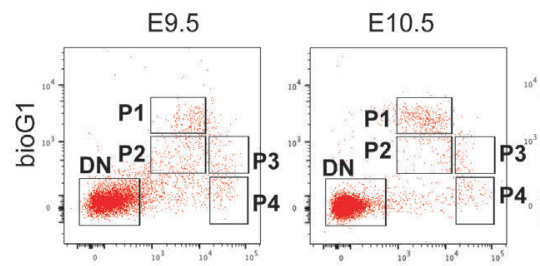

E11-11.5
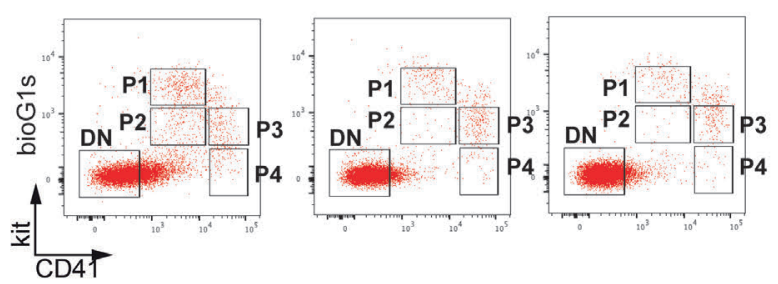

\section{$F$}

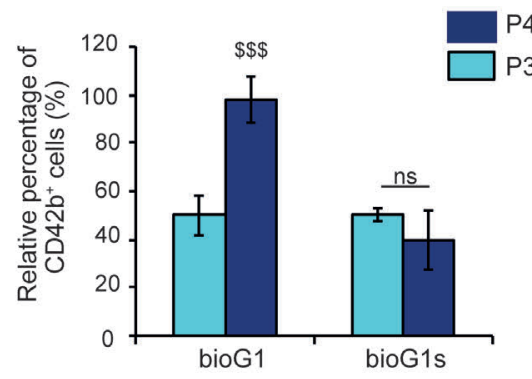

C

Number of Ter119+ cells Number of $\mathrm{Mac1}^{+}$and/or IYS $\mathrm{Gr} 1^{+}$cells / YS
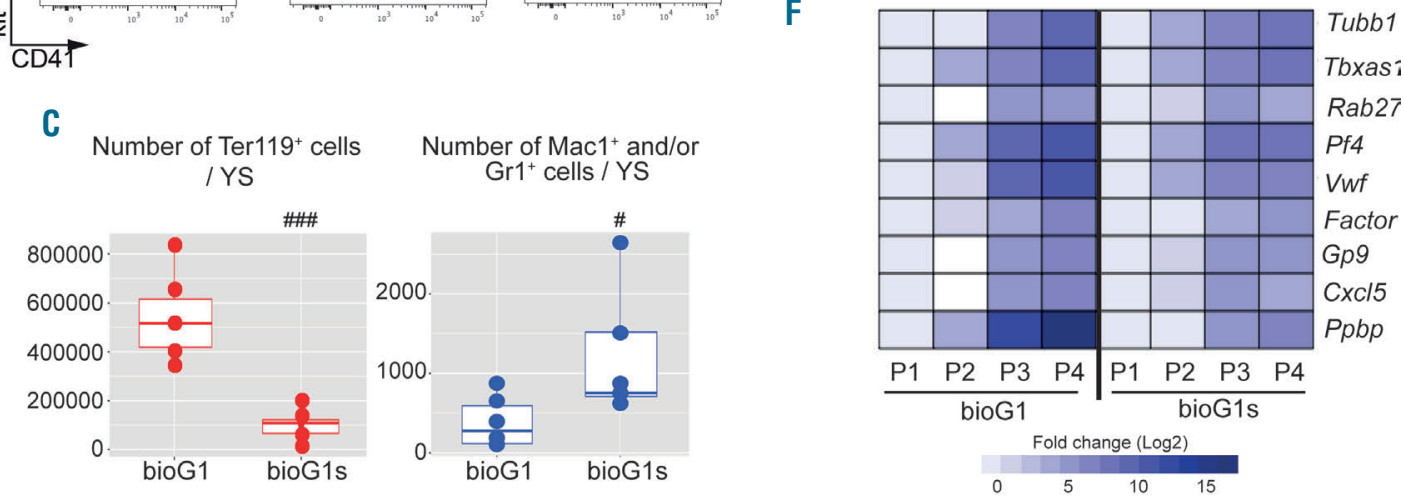

D

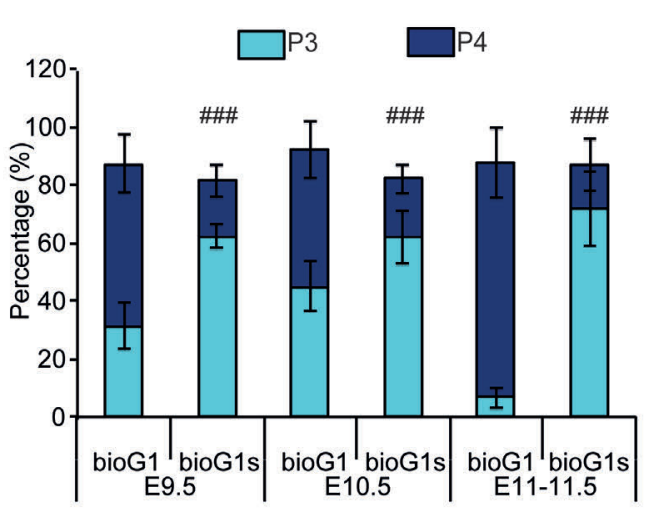

G

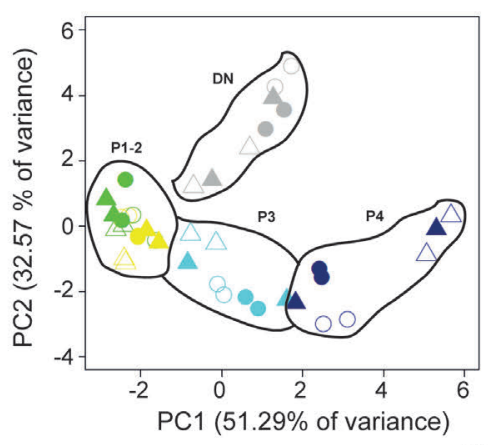

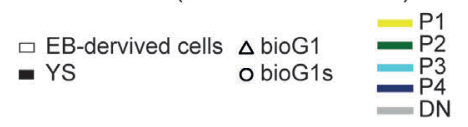

Figure 6. Hemopoietic populations in the yolk-sac. Expansion of GATA1s P3 relative to P4 populations. (A) Bar plot of absolute number (left) and percentage (right) of primitive progenitors with myeloid potential (CD16-CD32-) and definitive erythro-myeloid progenitors (CD16 ${ }^{+} \mathrm{CD} 32^{+}$) in day 6 (d6) EB-derived kit ${ }^{\text {ti }} \mathrm{CD} 41^{10}$ cells. Shown are mean percentage \pm 1 standard deviation (SD) from three independent experiments. Cell genotype is indicated. (B) Representative flow cytometry analysis plot of kit and CD41 expression from bioG1 (top) and bioG1s (bottom) at E9.5 (left), E10.5 (middle) and E11-11.5 (right) ( $n=5-7$ yolk sacs analyzed individually for each genotype). P1-P4 and DN populations indicated. (C) Box plot of absolute number/yolk sac of erythroid (left, Ter119+) and myeloid (right, Mac1 ${ }^{+}$and/or Gr1 ${ }^{+}$) in bioG1 and bioG1s. Each dot represents one yolk sac analyzed at E10.5 ( $n=5 /$ genotype). (D) Ratio of P3/P4 cells expressed as a percentage ( $y$-axis) in bioG1 and bioG1s yolk sacs at different time points ( $x$-axis). The ratios were calculated using the data shown in $B$, considering only the $C D 41^{\text {hi }}$ fraction. (E) Mean percentage $\pm 1 S D$ of CD42b+ E10.5 yolk sac cells in P3 and P4 in bioG1 and bioG1s ( $n=5$ for each genotype). (F) Heatmap of fold change of mRNA expression of megakaryocytic genes (rows) in E10.5 yolk sac cells purified from P1 to P4 from bioG1 (left) and bioG1s (right). Cells purified from two independent litters for each genotype, in two independent experiments. (G) Two dimensional principal component analysis (2D-PCA) plot of mRNA expression of 23 genes from either yolk sac cells (shaded symbols) or from embryoid bodies (EB)-derived in vitro cultures (open symbols), from bioG1 (triangles) and bioG1s (circles) genotypes, from P1 to P4 and double negative (DN) cells (colour coded as indicated below the figure). Conditions were performed in biological duplicates. Data taken from the Online Supplementary Figure S5F (EBderived cultures) and Online Supplementary Figure S6H (yolk sac). ${ }^{*} P<0.05$ and $* * * P<0.001$ between bioG1s and BirA. ${ }^{*} P<0.05$ and ${ }^{\# \# \#} P<0.001$ between bioG1s and bioG1. ${ }^{\$ \$ \$} P<0.01$ between P4 and P3. 
A

Day 6
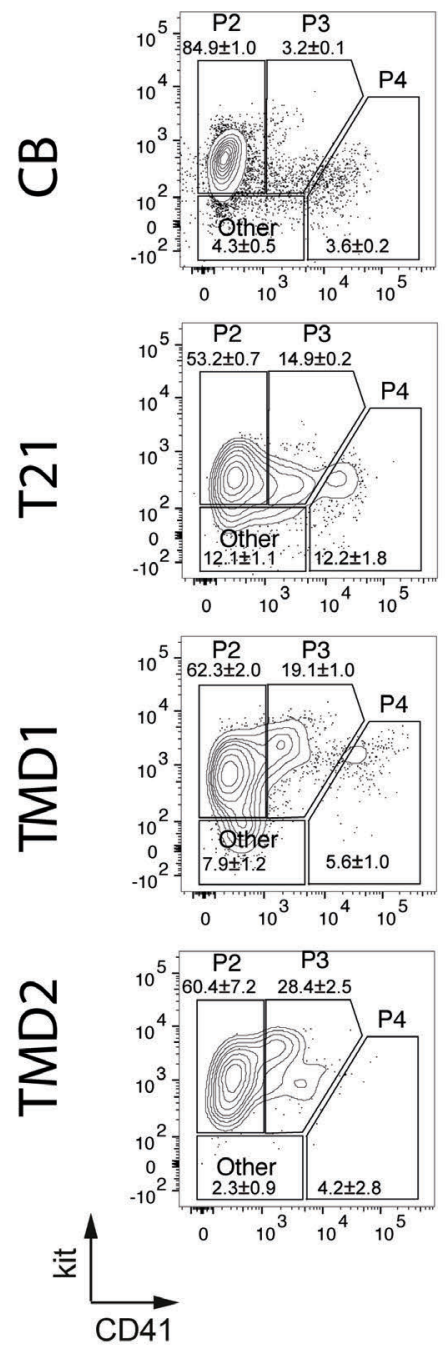

Day 9
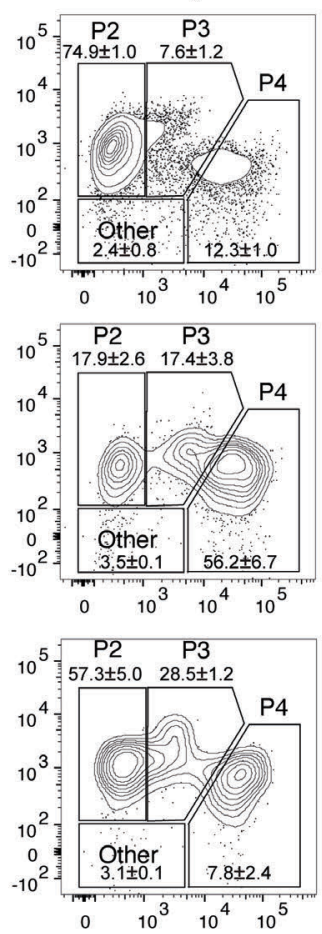

$10^{5} \begin{array}{cc}\mathrm{P} 2 & \mathrm{P} 3 \\ 38.9 \pm 12.8 & 23.8 \pm 8.8\end{array}$

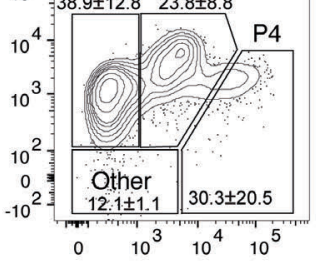

Day 12
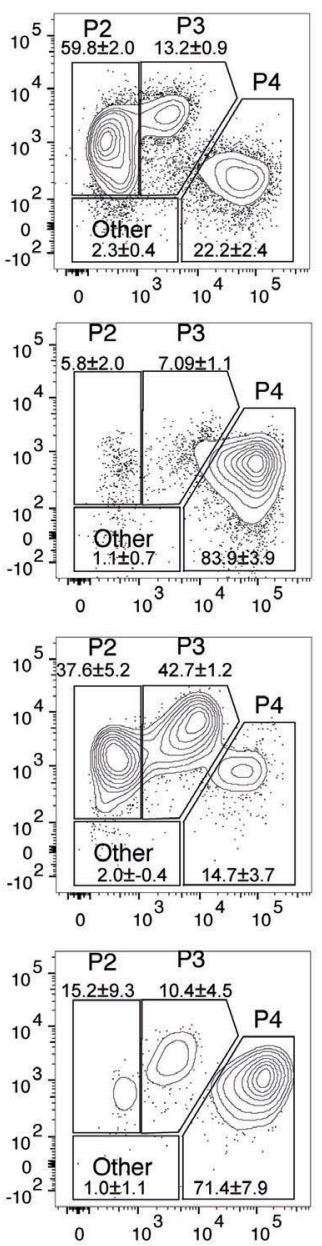

Day 15
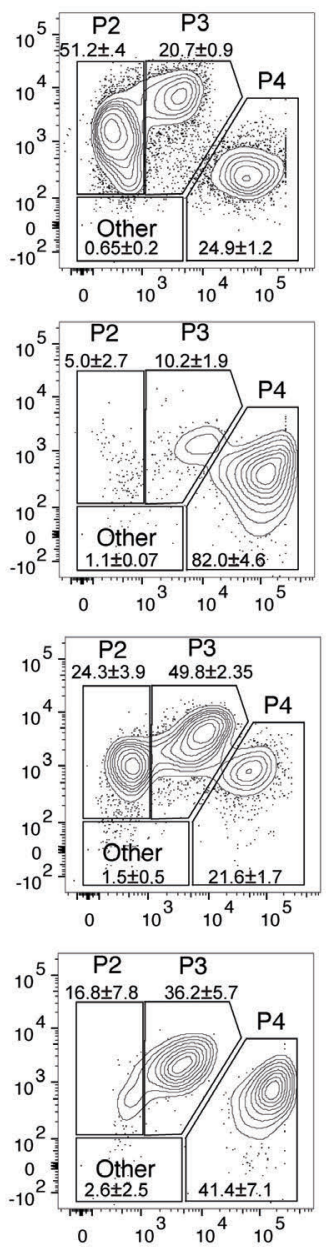

Day 15

B

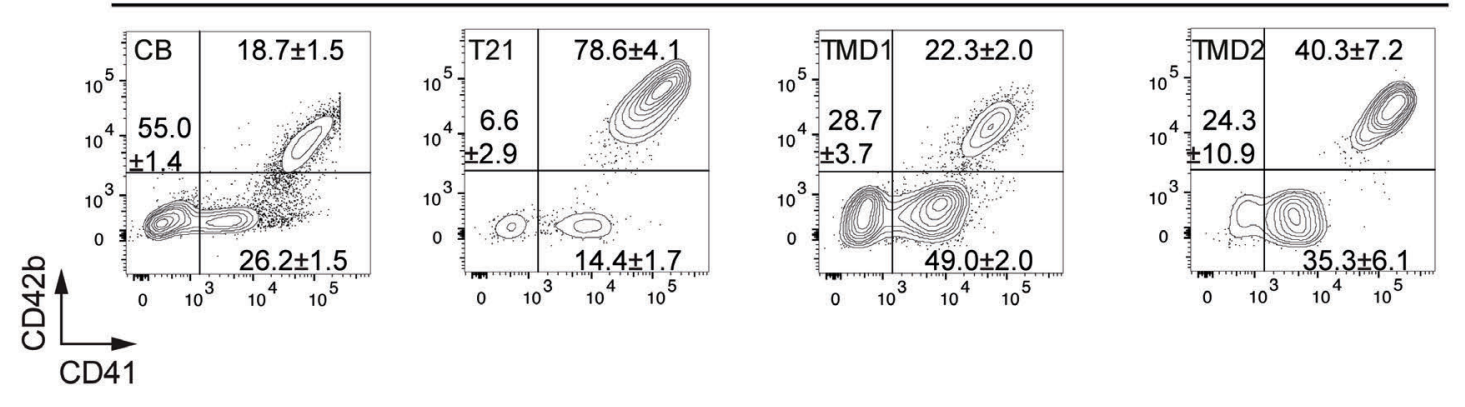

C

$\mathrm{CD}^{\mathrm{C}} 1^{+} / \mathrm{CD} 42 \mathrm{~b}^{+}$
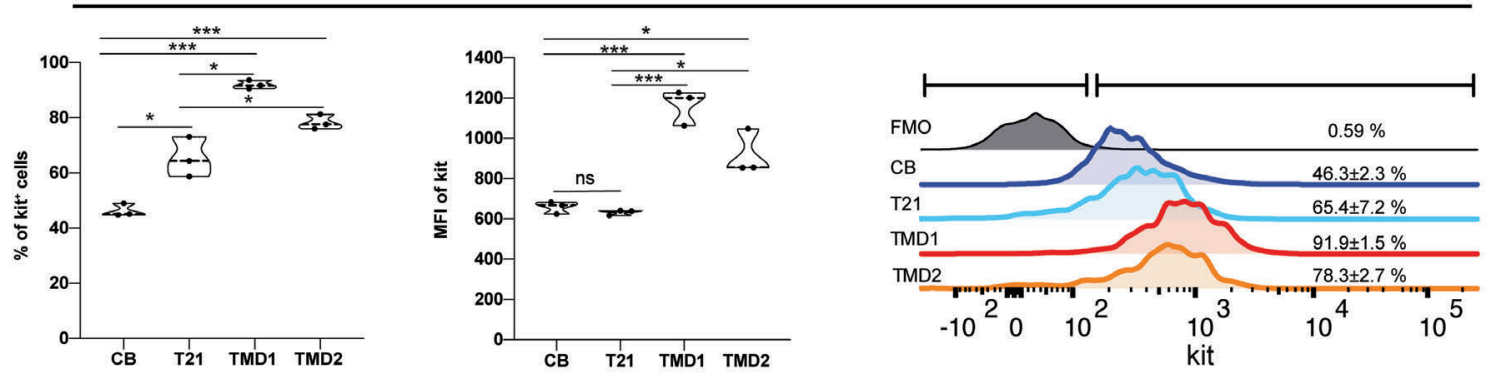

Figure 7. Legend on following page. 
Figure 7. (previous page) Megakaryocytic maturation defect is recapitulated in patients with transient myeloproliferative disorder. (A) Representative contour flow cytometry plots of live, CD235a negative cells at day 6 (d6), d9, d12 and d15 of megakaryocytic differentiation assessed by kit ( $y$-axis) and CD41 ( $\mathrm{x}$-axis) expression. CB refers to human neonatal GATA1 wild-type (WT) cells ; T21 refers to trisomy for chromosome 21 (T21) and GATA1 WT; TMD1 refers to T21 with a GATA1s mutation (exon2:c.G220C:p.V74L), variant allel frequency (VAF)=10.3\% of mononuclear cells (MNC); TMD2 refers to T21 with a GATA1s mutation (exon2:c.108_109del:p.S36fs ), VAF=9.2\% of MNC. Numbers within gates are the mean percentage \pm 1 standard devaitaion (SD) of cells from three culture experiments performed in parallel for each sample. (B) Representative contour plots of live, CD235a negative cells at d15 of megakaryocytic maturation assessed by CD42b ( $y$-axis) and CD41 (x-axis) expression for (from left to right) CB, T21, TMD1 and TMD2. Numbers within gates are as in A. (C) Plots comparing the percentage of kit positive cells (left), the mean fluorescence intensity (MFI) (middle) or both (right) in mature megakaryocytes defined as CD41 ${ }^{+} \mathrm{CD} 42 \mathrm{~b}^{+}$in the same set of samples. The unpaired $t$-test was used for statistical analysis. $* P<0.05, * * P<0.01$ and $P * * *<0.001$.

throid-myeloid progenitors (EMP). ${ }^{20}$ In d6 EB cultures, the majority of $\mathrm{kit}^{\mathrm{hi}} \mathrm{CD} 41^{\text {lo }}$ hemopoietic cells were $\mathrm{CD} 16^{+} \mathrm{CD} 32^{-}$primitive erythroid progenitors with myeloid potential (Figure 6A; Online Supplementary Figure $S 6 A-C)$ consistent with previous reports. ${ }^{20}$

Next, we analyzed hemopoietic cells from E9.5 to E11.5 yolk sac for kit and CD41 expression (Figure 6B). In E9.5 yolk sac, we identified populations with the same immunophenotypic profile as P1 to P4 and DN (kitCD41) populations seen in vitro in cultures, in both bioG1 and bioG1s embryos. From E9.5 to E11.5, the DN population was sustained in both bioG1 and bioG1s yolk sac. We then purified this population and quantitated the absolute number of cells/yolk sac (Figure 6C; Online Supplementary Figure S6D-F). Absolute numbers of DN cells were far lower in bioG1s yolk sac, with a significantly marked reduction in Ter119 $9^{+}$cells and increase in $\mathrm{Mac1}^{+} / \mathrm{Gr}^{+}$cells consistent with data from EB-derived cultures.

Turning to P1-P4 populations, there was a reduction of P1-P2 populations at E10.5, which virtually disappeared by E11-11.5 with mainly P3 and P4 populations present. Importantly, in bioG1s, there was a significant increase in $\mathrm{P} 3$ relative to $\mathrm{P} 4$ at each time point (E9.5-E11.5) and sustained higher levels of P3 cells at E11-11.5, mirroring in vitro culture data (Figure 6B, D). Purified P3 and P4 populations contained $\mathrm{CD}_{2} \mathrm{~b}^{+}$cells (Figure 6E; Online Supplementary Figure S6D, E, G). In control bioG1 cells there were significantly more CD $42 \mathrm{~b}^{+}$cells in $\mathrm{P} 4$ than P3, consistent with megakaryocyte maturation in $\mathrm{P} 4$. This was not the case in bioG1s P4 cells, consistent with aberrant, reduced megakaryocyte maturation. We also tested mRNA expression in purified E10.5 P1-P4 cells (Figure 6F). In bioG1 cells megakaryocytic gene expression (most noticeable for Ppbp, Vwf, Pf4, Tbxas1) increased progressively from P1/P2 to P3 then to P4. In contrast, in bioG1s cells expression of these genes did not increase from P3 to P4 cells, consistent with a megakaryocyte maturation defect. In order to ultimately confirm that the populations derived from the yolk sac were related to the ones identified from the EB model we performed a two-dimensional principal component analysis (PCA) (Figure 6G). Expression profiles of a panel of genes were interrogated by Fluidigm in DN and P1-P4 EB- and yolk sac-derived populations in both bioG1 and bioG1s (Online Supplementary Figures S5F, S6H). The genes were carefully selected for their well known role in specific hemopoietic lineages (Online Supplementary Table S2). The PCA was first performed on the EB population using prcomp function (PCA analysis using TRUE for the scale parameter). The yolk sac populations were then projected using the function predict. The most important finding was that yolk-sac and EB-derived DN and P1-P4 populations clustered together, consistent with the notion that the yolk sac and EB populations are transcriptionally similar. Principal component 1 (PC1) (51\% of variance) separated the P3 and P4 populations (genes whose expression contributed most to variance were the megakaryocyte genes - Tubb1, Factor V, Gp9 and Pf4) whereas PC2 (32\% of variance) separated the P1-P2 and DN populations (genes whose expression contributed most to variance were the erythroid genes - Klf1, Epor, and globin genes).

\section{Altered megakaryocytic differentiation is recapitulated in transient myeloproliferative disease samples}

Finally, we asked if the amplified immature megakaryocytic population observed in in vitro EB-derived Gata1s cells was also present in TMD patients (Online Supplementary Table S3). We analyzed the megakaryocytic differentiation of human cord blood $\mathrm{CD}_{3} 4^{+}$cells cultured in presence of thrombopoietin (TPO) and stem cell factor (SCF) (Figure 7; Online Supplementary Figure S7). Cells from disomic cord blood gave rise to $\mathrm{kit}^{\mathrm{hi}} \mathrm{CD} 41^{\mathrm{lo}} \mathrm{CD} 42 \mathrm{~b}^{-}$cells that then matured into a kit ${ }^{\mathrm{lo}} \mathrm{CD} 41{ }^{\mathrm{hi}} \mathrm{CD} 42 \mathrm{~b}^{+}$population (Figure 7A-B). Cells from T21 cord blood showed an exacerbated megakaryocytic differentiation, as most of the cells were kit ${ }^{\text {lo }} \mathrm{CD} 41^{\mathrm{hi}} \mathrm{CD} 42 \mathrm{~b}^{+}$by $\mathrm{d} 12$, in accordance with a previous report. ${ }^{21}$ Interestingly, cells derived from both TMD cord blood (harbouring a Gata1s mutation in around $10 \%$ of mononuclear cell [MNC]) showed an accumulation of the immature kit ${ }^{\text {hi }} \mathrm{CD} 41^{\text {lo }} \mathrm{CD} 42 \mathrm{~b}^{-}$population with a decreased maturation into $\mathrm{kit}^{\mathrm{lo}} \mathrm{CD} 41^{\mathrm{hi}} \mathrm{CD} 42 \mathrm{~b}^{+}$cells compared to T21-derived cells. Moreover, kit ${ }^{\text {lo }} \mathrm{CD} 41^{\text {hi }} \mathrm{CD} 42 \mathrm{~b}$ cells derived from both TMD samples harboured an increased level of kit expression compared to controls (Figure 7C), suggesting an altered megakaryocytic maturation.

\section{Discussion}

Our studies of a new knock-in GATA1s allele, in hemopoiesis from ESC and in murine yolk sacs, define the cellular mechanisms leading to a developmental-stage specific megakaryocyte myeloproliferation that likely contributes to the oncogenic effect of GATA1s. GATA1s results in a 10-fold increase in megakaryocytic cells from ESC cultures compared to control. Though prior work on GATA1s TMD-derived iPSC also demonstrated erythroid differentiation arrest and enhanced megakaryocyte differentiation, the stage in hemopoiesis where perturbed differentiation occurs was unclear. ${ }^{13}$ We now demonstrate that accumulation of megakaryocytic lineage cells occurs predominantly late in megakaryopoiesis, at an immature megakaryocyte precursor stage (where most cells are $2 \mathrm{~N}$ ), within a specific compartment (termed P3), characterized 
by high CD41 expression and low level of kit expression $\left(\mathrm{kit}^{\text {'o }} \mathrm{CD} 41^{\mathrm{hi}}{ }\right.$. Importantly, we showed the accumulation of a similar immature megakaryocytic population in samples from TMD patients, though its immunophenotype slightly differs between mouse and human.

A number of mechanisms may contribute to increased number of GATA1s P3 cells. Hemopoietic cells have a range of cell fate options including differentiation (with or without entering cell cycle), entering cell cycle without differentiating, apoptosis and quiescence. Our data shows that GATA1s P3 cells have increased number of cells in S-phase, reduced number in G0/G1 and a lower number of apoptotic cells compared to GATA1 P3 cells. Detailed kinetic studies of ES-derived hemopoiesis demonstrate a delay in exiting the P3 compartment into the next, more mature megakaryocyte compartment (termed P4) where cells have lost kit expression and presumably lost the proliferative drive afforded by kit signaling. For a 10-fold increase in cell number there need only just over three more cell divisions to account for the increase in GATA1s cell number.

Three major, open questions arise out of our work that provides a platform for future studies. The first two related questions are, what molecular mechanisms explain how GATA1s causes differentiation delay and why does differentiation delay specifically occur in the megakaryocyte lineage? Though the answers to these questions are unclear, prior data suggests that sustained elevated expression of GATA2 in GATA1s cells may play a role..$^{22}$ Chromatin occupancy by GATA2/E-box proteins/LMO2/FLI1/ERG/RUNX1 heralds megakaryocyte lineage priming and sustained GATA2 repression of specific loci is correlated with terminal megakaryocyte maturation ${ }^{23}$ and indirectly modulates megakaryocyte cell progression in GATA1 deficient megakaryocytes. ${ }^{24}$ However, proof that GATA2 is pivotal for GATA1s oncogenicity is still required and if GATA2 is needed, the mechanism by which it delays megakaryocyte differentiation in GATA1s cells requires further work.

Prior work has also suggested that GATA1 may directly interface with cell cycle..$^{25}$ Consistent with this, one report has shown that GATA1 directly binds $\mathrm{pRB/E2F2} \mathrm{via} \mathrm{amino}$ acid residues in the N-terminal GATA1 domain that is deleted in GATA1s. ${ }^{26}$ Normally, GATA1/pRB/E2F2 restrain uncommitted murine hemopoietic cell proliferation whereas GATA1s fails to bind pRB/E2F2 and fails to do this.

Our data also confirm that erythroid maturation is reduced in GATA1s cells consistent with prior work. ${ }^{13}$ One mechanism for this may be reduced GATA1s binding to cis-elements of erythroid genes which was demonstrated in an erythroid-megakaryocyte cell line mode ${ }^{27}$ that may cause a failure of terminal erythroid maturation, resulting in activation of an apoptotic program which is normally forestalled by GATA1 and erythropoietin. ${ }^{28}$

The third question is why does GATA1s exert a developmental-stage specific myeloproliferative effect? One possible explanation is that fetal liver-restricted IGF-1 signaling promotes E2F-induced erythro-megakaryocyte proliferation and that the extent of this proliferation is restrained by GATA1, but not GATA1s. ${ }^{29}$ Additionally, post-natal bone marrow-specific type 1 interferon signaling may actively suppress GATA1s megakaryocyte-erythroid progenitor growth promoting resolution of TMD in the post-natal period. ${ }^{30}$

In summary, our work now establishes the stage to test the role of previously identified molecular players (GATA1s, GATA2, E2F proteins, pRB, IGF-1 and interferon signaling) and possibly new determinants that regulate transition into and out of P3-like compartment in vivo and regulate the commitment of P2-like cells into either megakaryocytic or non-megakaryocytic paths of differentiation.

\section{Disclosures}

No conflicts of interest to disclose.

\section{Contributions}

GJ and NS conceived, designed and performed experiments, analyzed and interpreted the data, wrote the manuscript; HC and DCH performed experiments and analyzed the data; QC performed modeling analyses; $K S, B S, C G$ assisted with experiments; EK, MA generated ESC and mouse line bioG1; DW wrote the script to analyze staining data; GO performed computational analyses for RNAseq; JD, BU managed mouse colonies; $Q C, H C, D C H, B S$ and $D W$ contributed to editing the manuscript; $E M, I R, J S, C P$, and $P V$ designed the study, analyzed and interpreted the data, wrote the manuscript and academically drove the project.

\section{Funding}

$P V$ and IR are supported by Bloodwise Specialist Programme Grant 13001 and by the NIHR Oxford Biomedical Centre Research Fund. $P V$ and $C P$ are supported by programme grants from the MRC Molecular Haematology Unit (MC_UU_12009/11). CG is supported by a Wellcome Trust Clinical Training Fellowship.

\section{References}

1. Fujiwara Y, Browne CP, Cunniff K, Goff SC, Orkin SH. Arrested development of embryonic red cell precursors in mouse embryos lacking transcription factor GATA-1. Proc Natl Acad Sci U S A. 1996;93(22):1235512358.

2. Shivdasani RA, Fujiwara Y, McDevitt MA, Orkin SH. A lineage-selective knockout establishes the critical role of transcription factor GATA-1 in megakaryocyte growth and platelet development. EMBO J. 1997; 16(13):3965-3973.

3. Vyas P, Ault K, Jackson CW, Orkin SH, Shivdasani RA. Consequences of GATA-1 deficiency in megakaryocytes and platelets. Blood. 1999;93(9):2867-2875.

4. Wechsler J, Greene M, McDevitt MA, et al Acquired mutations in GATA1 in the megakaryoblastic leukemia of Down syndrome. Nat Genet. 2002;32(1):148-152.

5. Rainis L, Bercovich D, Strehl S, et al. Mutations in exon 2 of GATA1 are early events in megakaryocytic malignancies associated with trisomy 21. Blood. 2003; 102(3):981-986

6. Ahmed M, Sternberg A, Hall G, et al. Natural history of GATA1 mutations in Down syndrome. Blood. 2004;103(7):24802489.

7. Roberts I, Alford K, Hall G, et al. GATA1mutant clones are frequent and often unsuspected in babies with Down syndrome: identification of a population at risk of leukemia. Blood. 2013;122(24):3908-3917.

8. Yoshida K, Toki T, Okuno Y, et al. The landscape of somatic mutations in Down syndrome-related myeloid disorders. Nat Genet. 2013;45(11):1293-1299.

9. Labuhn M, Perkins K, Papaemmanuil E, et al. Mecanisms of progression of myeloid preleukemia to transformed myeloid leukemia in children with Down syndrome. Cancer Cell. 2019; 36(2):123-138.

10. Hollanda LM, Lima CS, Cunha AF, et al. An inherited mutation leading to production of only the short isoform of GATA-1 is associated with impaired erythropoiesis. Nat Genet. 2006;38(7):807-812.

11. Sankaran VG, Ghazvinian R, Do R, et al. Exome sequencing identifies GATA1 muta- 
Leukemic GATA1s delays megakaryocyte differentiation

tons resulting in Diamond-Blackfan anemia. J Chin Invest. 2012;122(7):2439-2443.

12. Li Z, Godinho FJ, Klusmann JH, GarrigaCant M, Yu C, Orin SH. Developmental stage-selective effect of somatically mutated leukemogenic transcription factor GATA1. Nat Genet. 2005;37(6):613-619.

13. Byrska-Bishop M, VanDorn D, Campbell $\mathrm{AE}$, et al. Pluripotent stem cells reveal trythroid-specific activities of the GATA1 Nterminus. J Chin Invest. 2015;125(3):9931005.

14. Hamlett I, Draper J, Strouboulis J, Iborra F, Porcher C, Ryas P. Characterization of megakaryocyte GATA1-interacting proteins: the corepressor ETO2 and GATA1 interact to regulate terminal megakaryocyte maturatron. Blood. 2008;112(7):2738-2749.

15. Driegen S, Ferreira R, van Zone A, et al. A generic tool for biotinylation of tagged proteens in transgenic mice. Transgenic Res. 2005;14(4):477-482.

16. Nishikii H, Etc K, Tamura $\mathrm{N}$, et al. Metalloproteinase regulation improves in vito generation of efficacious platelets from mouse embryonic stem cells. J Exp Med. 2008;205(8):1917-1927.

17. Paris J, Robertson S, Kennedy M, Wall C, Keller G. Development of erythroid and myeloid progenitors in the yolk sac and embryo proper of the mouse. Development. 1999;126(22):5073-5084

18. Keller G, Kennedy M, Papayannopoulou T,
Wiles MV. Hematopoietic commitment during embryonic stem cell differentiation in culture. Mol Cell Biol. 1993;13(1):473-486

19. Toper J, Koniski A, McGrath KE, et al. The megakaryocyte lineage originates from hemangioblast precursors and is an integral component both of primitive and of definetive hematopoiesis. Blood. 2007;109(4): 1433-1441.

20. McGrath KE, Frame JM, Fegan KH, et al. Distinct sources of hematopoietic progenitors emerge before HSCs and provide fundtonal blood cells in the mammalian embryo. Cell Rep. 2015;11(12):1892-1904

21. Roy A, Gowan C, Mead A, et al. Perturbation of fetal liver hematopoietic stem and progenitor cell development by risumy 21. Proc Natl Aced Sci U S A. 2012; 109(43):17579-17584.

22. Bourquin JP, Subramanian A, Langebrake $\mathrm{C}$, et al. Identification of distinct molecular phenotypes in acute megakaryoblastic leukemia by gene expression profiling. Proc Natl Accad Sci U S A. 2006;103(9): 3339-3344.

23. Pimkin M, Kossenkov AV, Mishra T, et al. Divergent functions of hematopoietic transcription factors in lineage priming and ifferentiation during erythro-megakaryopoiesis. Genome Res. 2014;24(12):19321944.

24. Huang Z, Dore LC, Li Z, et al. GATA-2 reinforces megakaryocyte development in the absence of GATA-1. Mol Cell Biol. 2009; 29(18):5168-5180.

25. Dubart A, Romeo PH, Vainchenker W, Dumenil D. Constitutive expression of GATA-1 interferes with the cell-cycle regulaion. Blood. 1996;87(9):3711-3721.

26. Kadi Z, Shimizu R, Ohneda O, et al. Direct binding of $\mathrm{pRb} / \mathrm{E} 2 \mathrm{~F}-2$ to GATA-1 regulates maturation and terminal cell division during erythropoiesis. PLoS Biol. 2009;7(6): e1000123.

27. Chilon TM, McNulty M, Goldenson B, Rosinski A, Crispino JD. Global transcriptome and chromatin occupancy analysis reveal the short isoform of GATA1 is deficlient for erythroid specification and gene expression. Haematological. 2015; 100(5):575-584.

28. Gregory T, Mu C, Ma A, Orin SH, Blobel GA, Weiss MJ. GATA-1 and erythropoietin cooperate to promote erythroid cell survival by regulating bcl-xL expression. Blood. 1999;94(1):87-96.

29. Klusmann JH, Godinho FJ, Heitmann K, et al. Developmental stage-specific interplay of GATA1 and IGF signaling in fetal megakaryopoiesis and leukemogenesis. Genes Lev. 2010;24(15):1659-1672.

30. Woo AJ, Wieland $\mathrm{K}$, Huang $\mathrm{H}$, et al. Developmental differences in IFN signaling affect GATA1s-induced megakaryocyte hyperproliferation. J Chin Invest. 2013; 123(8):3292-3304.

haematological | 2021; 106(4)

1119 